\title{
Development of Diagnostic Biomarkers for Detecting Diabetic Retinopathy at Early Stages Using Quantitative Proteomics
}

\author{
Jonghwa Jin, ${ }^{1,2}$ Hophil Min, ${ }^{1}$ Sang Jin Kim, ${ }^{3}$ Sohee Oh, ${ }^{4}$ Kyunggon Kim, ${ }^{1,2}$ \\ Hyeong Gon Yu, ${ }^{5}$ Taesung Park, ${ }^{6}$ and Youngsoo Kim ${ }^{1,2}$ \\ ${ }^{1}$ Department of Biomedical Sciences, Seoul National University College of Medicine, 28 Yongon-Dong, Seoul 110-799, Republic of Korea \\ ${ }^{2}$ Department of Biomedical Engineering, Seoul National University College of Medicine, 28 Yongon-Dong, \\ Seoul 110-799, Republic of Korea \\ ${ }^{3}$ Department of Ophthalmology, Samsung Medical Center, Sungkyunkwan University School of Medicine, \\ Seoul 135-710, Republic of Korea \\ ${ }^{4}$ Department of Biostatistics, Seoul Metropolitan Government-Seoul National University Boramae Medical Center, 20 Borame-ro 5-gil, \\ Dongjak-gu, Seoul 156-707, Republic of Korea \\ ${ }^{5}$ Department of Ophthalmology, Seoul National University College of Medicine, 28 Yongon-Dong, Seoul 110-799, Republic of Korea \\ ${ }^{6}$ Department of Statistics, Seoul National University, 1 Gwanak-ro, Gwanak-gu, Seoul 151-747, Republic of Korea
}

Correspondence should be addressed to Youngsoo Kim; biolab@snu.ac.kr

Received 10 November 2014; Accepted 5 March 2015

Academic Editor: Ying-Feng Zheng

Copyright (C) 2016 Jonghwa Jin et al. This is an open access article distributed under the Creative Commons Attribution License, which permits unrestricted use, distribution, and reproduction in any medium, provided the original work is properly cited.

Diabetic retinopathy (DR) is a common microvascular complication caused by diabetes mellitus (DM) and is a leading cause of vision impairment and loss among adults. Here, we performed a comprehensive proteomic analysis to discover biomarkers for DR. First, to identify biomarker candidates that are specifically expressed in human vitreous, we performed data-mining on both previously published DR-related studies and our experimental data; 96 proteins were then selected. To confirm and validate the selected biomarker candidates, candidates were selected, confirmed, and validated using plasma from diabetic patients without DR (No DR) and diabetics with mild or moderate nonproliferative diabetic retinopathy (Mi or Mo NPDR) using semiquantitative multiple reaction monitoring (SQ-MRM) and stable-isotope dilution multiple reaction monitoring (SID-MRM). Additionally, we performed a multiplex assay using 15 biomarker candidates identified in the SID-MRM analysis, which resulted in merged AUC values of 0.99 (No DR versus Mo NPDR) and 0.93 (No DR versus Mi and Mo NPDR). Although further validation with a larger sample size is needed, the 4-protein marker panel (APO4, C7, CLU, and ITIH2) could represent a useful multibiomarker model for detecting the early stages of DR.

\section{Introduction}

Diabetic retinopathy (DR) is a common microvascular complication caused by diabetes mellitus (DM) and is a leading cause of vision impairment and loss among adults [1]. It ultimately affects more than $90 \%$ of diabetic patients to some degree. Approximately $10 \%$ of patients with diabetes for $\geq 15$ years develop severe visual impairment, and about $2 \%$ of these patients become blind [2,3]. Vision impairment associated with DR can be further categorized into nonproliferative diabetic retinopathy (NPDR) and proliferative diabetic retinopathy (PDR); NPDR causes central vision loss when it induces diabetic macular edema (DME) [4].
PDR is the more advanced stage of DR and is characterized by retinal neovascularization. The manifestations of PDR include vitreous hemorrhage, formation of fibrous periretinal tissue accompanying neovascularization, traction retinal detachment, and, finally, total vision loss $[5,6]$. Currently, the main treatments for DR are retinal photocoagulation and vitreoretinal surgery; there are no specific drugs currently available to prevent or slow down DR. Although the control of systemic risk factors, such as hyperglycemia, hypertension, and dyslipidemia [7], has been shown to improve clinical therapies for diabetes-induced vision loss, more effective clinical therapies for DR patients are needed. 
Recently, biomarker discovery has become important in many aspects of disease diagnosis and drug discovery and development [8-10]. Predictive biomarkers can save time and money and lead to better diagnoses, ultimately aiding the cure of diseases. Indeed, many biomarkers have an important diagnostic role in the detection or prediction of disease. However, convenient biomarkers must be detectable in tests of blood, urine, or saliva, which can be performed at the bedside or in an outpatient setting [11-13]. Although urinary and tissue samples have been primarily used in the past to identify biomarkers, plasma and serum can also be collected noninvasively and contain factors that can be more effective in biomarker development. Indeed, blood is routinely collected and contains thousands of proteins, including secreted proteins and proteins shed into the blood by tumors $[9,14-16]$.

The pipeline for biomarker development usually includes 4 steps-discovery, verification, validation, and product development. Clinical verification or validation is well known to be the biggest barrier for biomarker development. To overcome this barrier, effective methods to facilitate largescale biomarker validation are needed. Immunological methods and multiple reaction monitoring (MRM) are being considered as possible solutions. Between these 2 methods, the MRM approach has a much higher throughput and accuracy and allows for substantial multiplexing [17]. With the use of MRM analysis, more than 100 candidate proteins can be simultaneously targeted and measured at the clinical verification stage.

There have been many proteomic studies on DR that analyze the retina, vitreous, or plasma, which have led to a better understanding of the DR proteome [17-19]. Based on these studies, we decided to use a systemic approach to identify biomarkers for DR-related diseases.

First, to obtain biomarker candidates that are specifically expressed in the tissues of macula hole $(\mathrm{MH}$, nondiabetic controls), NPDR, and PDR patients, we performed datamining on the previously published DR-related studies $[6,18-$ 25 ] and our experimental data set in human vitreous. This proteomic information provided baseline data, including 96 possible DR biomarker candidates. Second, the 96 selected candidates were used to carry out a semiquantitative multiple reaction monitoring (SQ-MRM) verification experiment, in which we used plasma samples from type 2 diabetes patients without DR (No DR), with mild nonproliferative DR (Mi NPDR), and with moderate nonproliferative DR (Mo NPDR). We were able to select 15 candidates by SQ-MRM verification and, thereafter, subsequent stable-isotope dilution multiple reaction monitoring (SID-MRM) verification narrowed these candidates down to 11 potential markers. Third, we constructed a model for a multimarker panel using these 11 verified markers and performed leave-one-out cross validation (LOOCV) on the panel to avoid overfitting and to calculate error rates. For this statistical evaluation, we used linear discriminant analysis (LDA) methods.

Consequently, this systemic approach allowed us to select optimal biomarker candidates for inclusion in a multimarker panel. We determined that a 4-marker panel was a better diagnostic tool for detecting early grade NPDR and was associated with lower error rates than the use of the single best marker alone.

\section{Materials and Methods}

2.1. Plasma Sample Preparation. To verify DR biomarker candidates, we collected plasma from 20 diabetic patients with no diabetic retinopathy (No DR), mild nonproliferative diabetic retinopathy (Mi NPDR), or moderate nonproliferative diabetic retinopathy (Mo NPDR). DR was classified by the international clinical diabetic retinopathy disease severity scale [26]. Each plasma sample was collected in $10 \mathrm{~mL}$ tubes containing $\mathrm{K}_{2}$-EDTA. Tubes were centrifuged at $3000 \times \mathrm{g}$ for $10 \mathrm{~min}$ at $4^{\circ} \mathrm{C}$, and $100 \mu \mathrm{L}$ was aliquoted into new tubes and stored at $-70^{\circ} \mathrm{C}$.

All patients provided informed consent before being enrolled in this study, in accordance with the protocol approved by the Institutional Review Board at Seoul National University Hospital (IRB number H-0807-086-251).

2.2. MARS Depletion. To deplete plasma using a MARS column, plasma was diluted 5-fold with MARS buffer A (1:4) and filtered with a $0.22 \mu \mathrm{m}$ Ultrafree-MC Durapore centrifugal filter (Cat. number UFC30GVNB, Millipore, Billerica, MA, USA). Each plasma sample was applied to a MARS column on an LC-10AT HPLC system (Shimadzu, Kyoto, Japan). The sample loop volume of the HPLC was set to $200 \mu \mathrm{L}$, and $200 \mu \mathrm{L}$ of 5 -fold diluted plasma was injected into the MARS column. The total LC run time of $37 \mathrm{~min}$ involved the following: $100 \%$ MARS buffer $\mathrm{A}$ at a flow rate of $0.7 \mathrm{~mL} / \mathrm{min}$ for the first $11 \mathrm{~min}$, sample injection, wash for $11 \mathrm{~min}, 100 \%$ MARS buffer $\mathrm{B}$ at a flow rate of $1.0 \mathrm{~mL} / \mathrm{min}$ for $5 \mathrm{~min}$, and $100 \%$ MARS buffer $\mathrm{A}$ at a flow rate of $0.7 \mathrm{~mL} / \mathrm{min}$ for $10 \mathrm{~min}$.

The UV detector was set to $280 \mathrm{~nm}$ for plasma injection, and the eluted fractions were collected in $250 \mu \mathrm{L}$ aliquots. The flow-through and bound fractions were eluted in 10 fractions (total, $2.5 \mathrm{~mL}$ ), and the eluted fractions were pooled for MRM.

2.3. Preparation of Plasma for Mass Spectrometry. The protein concentration in the vitreous and depleted plasma was measured using BCA methods according to the protocol provided by the manufacturer. Aliquots of $100 \mu \mathrm{g}$ protein were reduced, alkylated, and digested according to the protocol. Briefly, $60 \mu \mathrm{L} 9 \mathrm{M}$ urea (5.4054 g) and $30 \mathrm{mM}$ dithiothreitol (DTT; $0.04628 \mathrm{~g}$ ) in $10 \mathrm{~mL} 100 \mathrm{mM}$ Tris-base ( $\mathrm{pH} 8.0$ ) were added to each sample and incubated for $30 \mathrm{~min}$ at $37^{\circ} \mathrm{C}$. Each sample was allowed to cool at room temperature before $9 \mu \mathrm{L} 500 \mathrm{mM}$ iodoacetamide (IAA; $0.0925 \mathrm{~g} / \mathrm{mL}$ ) was added. The solution was incubated for $20 \mathrm{~min}$ at room temperature. To dilute the urea from $6 \mathrm{M}$ to $0.6 \mathrm{M}, 771 \mu \mathrm{L} 100 \mathrm{mM}$ Tris buffer ( $\mathrm{pH} 8.0$ ) was added to each sample. Each sample was then digested with trypsin (Promega, Madison, WI, USA) at a proteinto-enzyme ratio of $50: 1$ at $37^{\circ} \mathrm{C}$ overnight. To quench the digestion reaction, $50 \mu \mathrm{L} 0.1 \%$ TFA was added. The digested peptide mixture was applied onto an HLB Oasis cartridge (Waters Milford, MA, USA) for desalting, and peptides were eluted using $1 \mathrm{~mL} 60 \%$ ACN with $0.1 \%$ FA solution. 
2.4. Determination of Transitions Using the Skyline Program. To determine the optimal MRM transition, including precursor $(\mathrm{Q} 1)$ and fragment $(\mathrm{Q} 3)$ ions, we used an in silico method utilizing the Skyline program (version 2.1), which is an open-source software application for developing MRM methods and analyzing MRM data [27]. The FASTA file for each protein selected by data-mining was imported into the Skyline program; the precursor and fragment ions for each protein were generated by performing in silico digestion. Our peptide filter conditions were as follows: the precursor length range was set at 8 to 20 amino acids, and peptides with repeat arginine (Arg, R) or lysine (Lys, K) residues were not used. To avoid using peptides that included potentially modified forms in the sequence, methionine (Met, M) and cysteine (Cys, C) residues were discarded. If proline (Pro) was next to an arginine (Arg, R) or lysine (Lys, $\mathrm{K}$ ) residue, the peptide was not used. Peptides containing histidine $(\mathrm{His}, \mathrm{H})$ were also discarded because of the possibility of alterations in the side chain charge.

2.5. Multiple Reaction Monitoring Using Triple Quadrupole Mass Spectrometry. Our semiquantitative multiple reaction monitoring (SQ-MRM) and stable-isotope dilution multiple reaction monitoring (SID-MRM) analyses were performed using a triple quadrupole linear ion trap mass spectrometer (a 4000 QTRAP coupled with a nano-Tempo MDLC, Applied Biosystems, Carlsbad, CA, USA), as described in a previous study [28]. To reduce the void volume and obtain sharper intensity peaks, we used a modified sample loop $(100 \mu \mathrm{m}$ ID capillary tubing containing $1.0 \mu \mathrm{L}$ sample) in an autosampler. In our MRM analysis, a $15 \mathrm{~cm}$ homemade analytical column was used-an IntegraFrit capillary (ID, $75 \mu \mathrm{m}$; OD, $360 \mu \mathrm{m})$ was packed with Magic C18AQ (200 A, Michrom Bioresources, Madison, WI, USA). We directly injected $1 \mu \mathrm{L}$ of our prepared sample with Sol A (98\% DW, $2 \% \mathrm{ACN}$, and $0.1 \% \mathrm{FA})$ and Sol B (98\% ACN, 2\% DW, and $0.1 \% \mathrm{FA})$ into the analytical column without a trap column; the flow rate was set to $300 \mathrm{~nL} / \mathrm{min}$. Exponential gradient elution $(60 \mathrm{~min})$ was performed by increasing the mobile phase composition from 0 to $40 \%$ Sol B. The gradient was then increased to $90 \%$ $\mathrm{B}$ for $10 \mathrm{~min}$ and $0 \% \mathrm{~B}$ for $10 \mathrm{~min}$ to equilibrate the column for the next run. The optimal parameters for a triple quadrupole mass spectrometer that interfaced with a nanospray source were set as follows: ion spray (IS), $2100 \mathrm{~V}$; source temperature, $160^{\circ} \mathrm{C}$; high collision gas, approximately $4-3 \times 10^{-5}$ torr; and curtain gas, 15 . MS parameters for declustering potential (DP) and collision energy (CE) were determined using the Skyline program. In the MRM run, the scan time for each transition and the pause time between transition scans were set to $15 \mathrm{~ms}$ and $5 \mathrm{~ms}$, respectively. MRM analysis was composed of 2 steps: the first MRM step determined the transition and the second step monitored the target transition for quantification. In the first MRM step, an enhanced mass scan (EMS) ranging from 400 to $1200 \mathrm{~m} / z$ (scan speed, $4000 \mathrm{Da} / \mathrm{s}$ ) was performed, and an enhanced product ion (EPI) scan was then carried out to obtain MS/MS spectra. A MASCOT search was performed to identify the protein. In the second MRM step, an MRM analysis was performed to quantify the selected target transition without any information-dependent scan or MS/MS scan (see the Supplementary Methods for more details in Supplementary Material available online at http://dx.doi.org/10.1155/2015/6571976). In the SQ-MRM analysis, we spiked each sample with $50 \mathrm{fmol}$ betaGAL (GDFQFNISR, 542.3/636.3 $\mathrm{m} / \mathrm{z}$ ) during our preparation of the digested sample. In the SID-MRM analysis, 15 synthetic heavy peptides ( $>95 \%$ purity) were used.

2.6. Synthetic Peptides. For the SID-MRM analysis, we first synthesized stable-isotope standard (SIS) peptides ( $>95 \%$ purity) for 15 proteins (APLP2, APOA4, APOH, B3GNT1, C4B, C5, C7, CD14, CLU, FN1, GSN, ITIH2, KRT1, SERPINF1, and VTN). Synthetic peptides were obtained from 21st Century Biochemicals (Marlboro, MA, USA). Peptide sequences were synthesized as unmodified peptides with free $\mathrm{N}$ - and C-terminal amino acids. The purity of the synthetic peptides was $>95 \%$, as measured by HPLC. For stable isotope-labeled peptides, the C-terminal arginine or lysine contained ${ }^{13} \mathrm{C}$ and ${ }^{15} \mathrm{~N}$-labeled atoms. Peptide stock concentrations were determined by amino acid analysis (AAA).

2.7. Determination of the Lowest Limit of Detection (LLOD) and the Lowest Limit of Quantification (LLOQ). To determine the LLOD and LLOQ, we used the method described by Keshishian et al. and other authors [29-31]. The LLOD was calculated based on the variance of the blank sample (sample b, with no "spiked" in analyte) and standard deviation (S.D.) of the sample with the lowest concentration among the "spiked" in samples $(0.1 \mathrm{fmol} / \mu \mathrm{L})$. Equation (1) was used to determine LLOD as follows:

$$
\mathrm{LLOD}=\mathrm{LOB}+c_{\beta} \times \text { S.D.s. }
$$

For this equation, if the analyte is detected when it is not present and if the analyte is not detected when it is present, the type I error rate $\alpha$ and the type II error rate $\beta$ are 0.05 and 0.05 , respectively. The limit of the blank (LOB) was defined as the 95th percentile of the blank sample. For a relatively small number of repeated measurements of the blank, the $c_{\beta}$ was approximated as follows: $c_{\beta}=t_{1-\beta}$; thus,

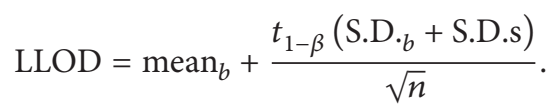

For this equation, $t_{1-\beta}$ is equal to the $(1-\beta)$ percentile of the standard $t$ distribution with $n$ degrees of freedom; $n$ is equal to the number of replicates. Consequently, the LLOD equation can be expressed as in the following equation:

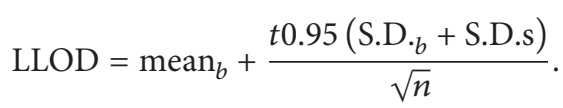

The LLOQ was calculated using the following standard calculation [29-31]: LLOD $\times 3=$ LLOQ.

2.8. Statistical Analyses. To enhance the classification power of the biomarkers, we constructed a multimarker panel using individual biomarkers and then performed a statistical evaluation of the panel. Using the SPSS Statistics software program 


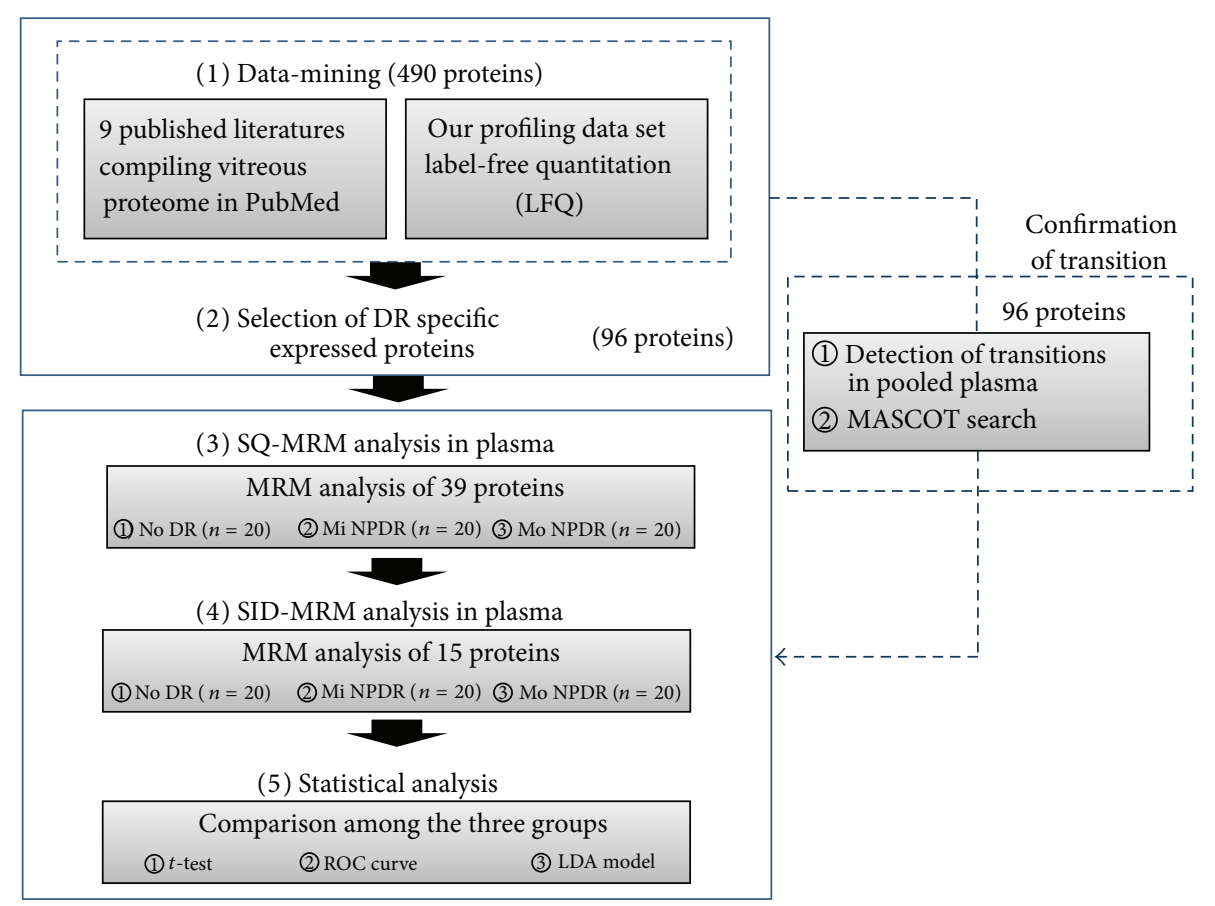

FIGURE 1: The overall scheme for DR biomarker development. The first step to develop biomarkers is to identify candidates. To that end, we performed a comprehensive proteomics study of DR samples, which included 2 steps: (1) discovering and (2) validating biomarker candidates. In the discovery stage, 96 proteins were selected as DR-specific proteins in human vitreous. Using a MASCOT search, 39 proteins were confirmed in human plasma. In the verification stage, SQ-MRM and SID-MRM analyses were performed to verify the candidates. Finally, statistical analysis was performed on the 15 verified candidates.

(IBM Inc., Armonk, NY, USA, ver. 20), we first conducted a correlation analysis to examine the variable stability by Pearson's correlation analysis. Second, Student's $t$-test and stepwise multivariate analysis of variance (MANOVA) analysis [32] were performed to select the multimarker proteins that contributed to the discriminatory power between the No DR and Mo NPDR groups. Finally, to validate the multimarker models from multivariate analysis, LOOCV was conducted to avoid overfitting and to calculate the error rate of the model by comparing the No DR and Mo NPDR groups. To validate the multimarker panel, we used the linear discriminant analysis (LDA) method. We used the MedCalc (MedCalc Software, Mariakerke, Belgium) program to perform the ANOVA and chi-square tests and to generate receiver operating characteristic (ROC) curves.

2.9. Gene Ontology (GO) and Functional Analysis. To analyze the GO terms in the protein data sets, we used the DAVID bioinformatics resource (http://david.abcc.ncifcrf.gov/), which allows both the functional classification and the ID conversion of the proteins that we identified. The "biological process" and "molecular function" classifications were analyzed using PANTHER ID numbers (http://www.pantherdb.org/).

\section{Results}

3.1. Plasma Characteristics of the No DR, Mi NPDR, and Mo $N P D R$ Groups. We collected 20 plasma samples with a $1: 1$ gender ratio from each of the No DR, Mi NPDR, and Mo NPDR patient groups. The patient gender, patient age, DM status, HbAlc, and plasma concentration for these 60 plasma samples are shown in Supplementary Table 1. We sorted patients that had no complications from diabetes except diabetic retinopathy and, thus, all patients in this study have no kidney diseases like diabetic nephropathy.

To examine whether any other factors could interfere with the MRM analysis, we carried out ANOVA tests for age, DM duration, and HbAlc and used chi-square tests for gender and hypertension (Supplementary Table 2). We detected no significant differences related to gender, age, hypertension, or HbAlc. For DM duration, increased DM duration was observed in the NPDR group compared to the No DR group. There were also differences in DM duration between the No DR and Mo NPDR groups, as indicated by the ANOVA findings (F-statistics).

3.2. Our Overall Scheme for DR Biomarker Development. The first step in biomarker development is to identify candidates. To this end, we performed a comprehensive proteomics study of DR samples, which included 2 steps: (1) discovery of biomarker candidates in vitreous samples and (2) verification of biomarker candidates in plasma samples (Figure 1).

First, we performed data-mining on previously published DR-related studies and our experimental data from human vitreous samples. In the candidate discovery stage, 96 proteins were selected from literatures and our experimental data (Supplementary Tables 3-5). The 1870 transitions for these 96 
proteins were generated using the Skyline program and then were confirmed by MRM analysis, where 39 proteins were confirmed (Supplementary Table 6). The 39 candidates were then examined by SQ-MRM analysis, where the expression of 15 proteins significantly differed between the No DR and $\mathrm{Mi}$ NPDR or Mo NPDR groups with an AUC > 0.7. Furthermore, the 15 candidate proteins were also verified by SID-MRM analysis using the same plasma samples.

For the final stage of biomarker development, models were constructed for multimarker panels composed of protein variables having discriminatory power. First, we checked for correlation and multicollinearity among the 15 potential markers to evaluate the discrimination power. Next, using Student's $t$-test and stepwise MANOVA analyses, we selected 4 potential markers with an expression pattern that differed significantly between the No DR and Mo NPDR groups. Using the 4 differentially expressed marker proteins, a multimarker panel was constructed and statistical validation was performed using LOOCV. To examine the efficacy of the model, the LDA method was used for statistical analysis (Figure 1).

3.3. Selection of Biomarker Candidates. We carried out datamining on all previously published DR-related studies in humans [6, 18-25] (Supplementary Table 5). For standardization and integration of protein names, all accession number formats, such as Uniprot, IPI, GI number, and GenBank number, were converted into gene symbols. Protein data, such as identification and quantification in a DR-related study, were extracted; data were compiled from 9 DR proteomics studies that reported quantitative data, and a total of 465 proteins were collected by data-mining. Additionally, we also collected LC-ESI-MS/MS experimental data, 136 differentially expressed proteins. In the data-mining stage, 490 proteins were ultimately identified. To select the most reliable candidates, proteins found in more than 4 hits in 10 datasets were selected (our experimental data set had " 2 weighted hits"). Consequently, 96 proteins were finally selected as candidates and 1870 transitions were generated for these proteins using the Skyline program.

For MRM analysis, we first optimized the collision energy (CE) and declustering potential (DP) using the Skyline parameter optimization module. We then confirmed whether the 1870 transitions could be detected in pooled plasma and also tested whether these transitions could be identified as target proteins using a MASCOT search. From MASCOT searches, 39 proteins were ultimately confirmed, for which 22 proteins had 2 peptides and 17 proteins had 1 peptide (Supplementary Table 6).

3.4. Confirmation of Biomarker Candidates by SQ-MRM Analysis. Before performing MRM analysis, we first determined the MRM reproducibility in 3 replicate experiments using pooled plasma samples for the detection of 15 representative proteins (Figure 2). We found that APLP2, FN1, ITIH2, and SERPINF1 had higher CV values $(19 \%, 14 \%, 18 \%$, and $13 \%$, resp.), while the other proteins had lower CV values (Figure 2). However, the CV values for all of the proteins were less than $20 \%$, even though we did not use an internal standard for normalization.

To obtain a stable quantitative peak area in the SQMRM analysis, we spiked $50 \mathrm{fmol}$ betaGAL (GDFQFNISR, $542.3 / 636.3 \mathrm{~m} / \mathrm{z}$ ) into the individual digested samples. To verify the selected biomarker candidates, we measured the relative concentration of the 39 candidates in the No DR, Mi NPDR, and Mo NPDR groups. Using MRM analysis, we confirmed differential measurements of these proteins among the No DR $(n=20)$, Mi NPDR $(n=20)$, and Mo NPDR $(n=20)$ groups.

In our quantification analysis (Figure 3), 15 proteins (amyloid-like protein 2, APLP2; apolipoprotein A-IV, APOA4; beta-2-glycoprotein 1, APOH; N-acetyllactosaminide beta-1,3-N-acetylglucosaminyltransferase, B3GNT1; complements C4-B, C4B; complements C5, C5; complement components $\mathrm{C}$, $\mathrm{C} 7$; monocyte differentiation antigens CD14, CD14; clusterin, CLU; fibronectin, FN1; gelsolin, GSN; inter-alpha-trypsin inhibitor heavy chain $\mathrm{H} 2$, ITIH2; keratin type II cytoskeletal 1, KRT1; pigment epithelium-derived factor, SERPINF1; and vitronectin, VTN) were significantly differentially expressed either in the No DR versus Mi NPDR (AUC value $>0.7$ ) or in the No DR versus Mo NPDR (AUC value $>0.7)$ comparisons. Notably, B3GNT1 (0.88) and both C7 and ITIH2 (0.85) showed the highest AUC values in the No DR versus Mi NPDR and the No DR versus Mo NPDR comparisons, respectively. We performed MRM verification using SID-MRM on the 15 selected candidate proteins associated with an AUC value $>0.7$.

3.5. Functional Analysis of the 15 Verified Proteins. To examine the biological functions of the 15 proteins from the SQMRM analysis, proteins were assigned biological process and molecular function categories using the PANTHER classification program. The largest assigned biological process and molecular function category were "immunity and defense" (33\% and 20\%, resp.) (Supplementary Figure 1A). Moreover, 5 proteins $(\mathrm{APOH}, \mathrm{C} 4 \mathrm{~B}, \mathrm{C} 5, \mathrm{C} 7$, and $\mathrm{CD} 14)$, which are involved in biological process subcategories of "immunity and defense," all showed a downregulated pattern (100\%) when the No DR group was compared to the Mo NPDR group. Two proteins (APLP2 and KRT1), which are related to the biological process subcategory "developmental process," also showed a downregulated pattern $(100 \%)$ compared to the No DR and Mo NPDR groups.

To investigate the signaling pathways related to the 15 verified proteins, we conducted a signaling pathway analysis. We identified 2 signaling pathways (the actin cytoskeleton and complement cascade pathways) associated with these proteins in the KEGG database (Supplementary Figures 1B and C). CD14, FN1, and GSN play roles in the actin cytoskeleton pathway; CD14 and GSN were downregulated, whereas FN1 was upregulated. $\mathrm{C} 4 \mathrm{~B}, \mathrm{C} 5$, and $\mathrm{C} 7$ play roles in the complement cascade pathway; all 3 proteins were downregulated.

3.6. The Verification of the 15 Candidate Proteins Using SIDMRM Analysis. Before we synthesized the SIS heavy peptides for SID-MRM analysis, we first examined how many peptides 
APLP2

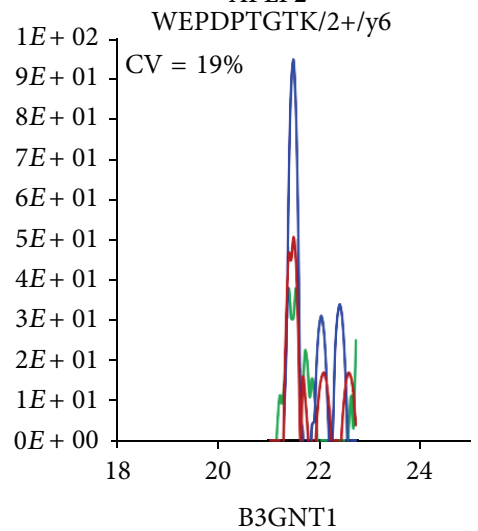

QYGFNR/2+/y5
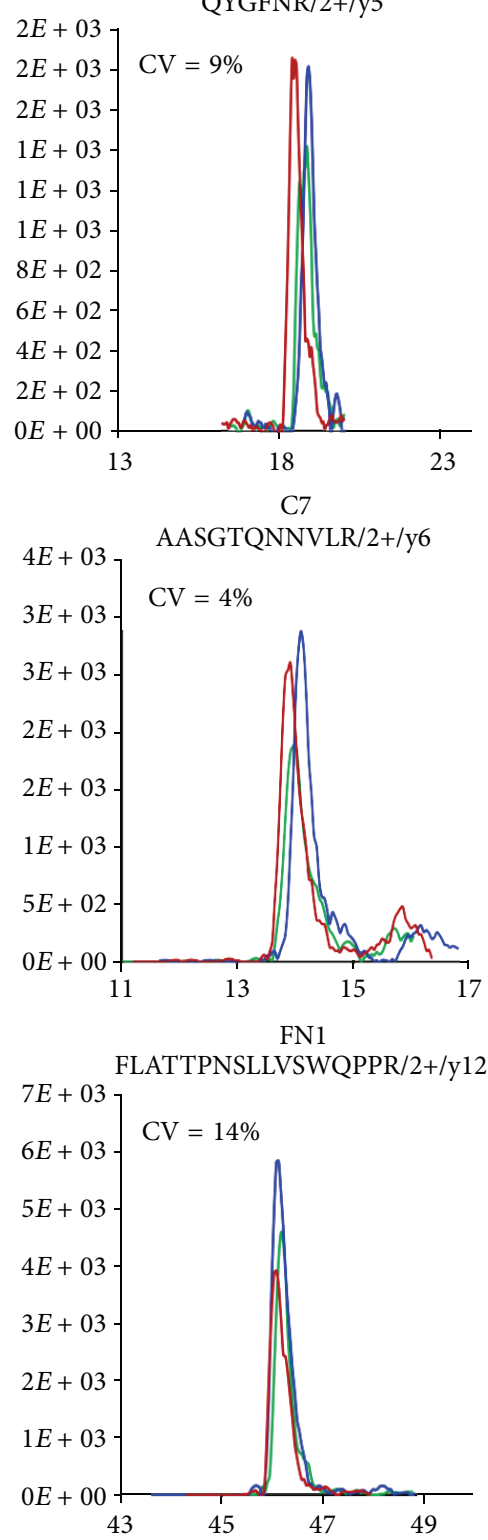

APOA4

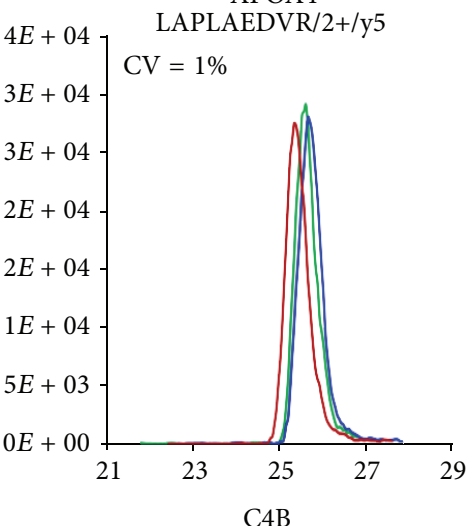

$5 E+04 \quad$ VDFTLSSER/2+/y 6

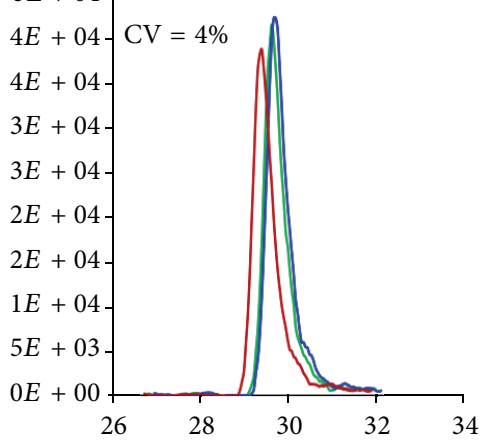

CD14

FPAIQNLALR/2+/y6

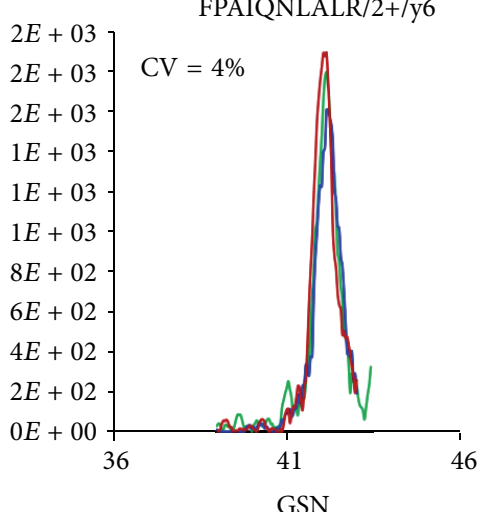

GGVASGFK/2+/y5

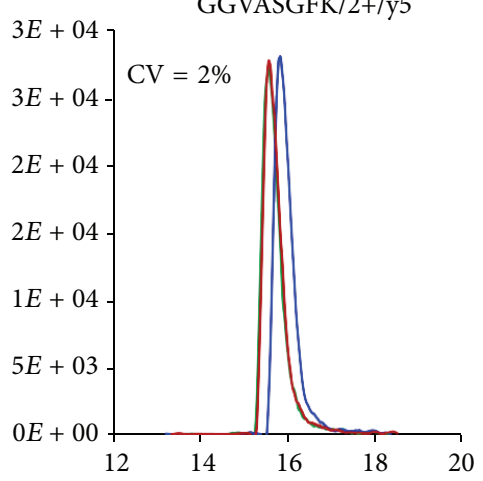

$\mathrm{APOH}$
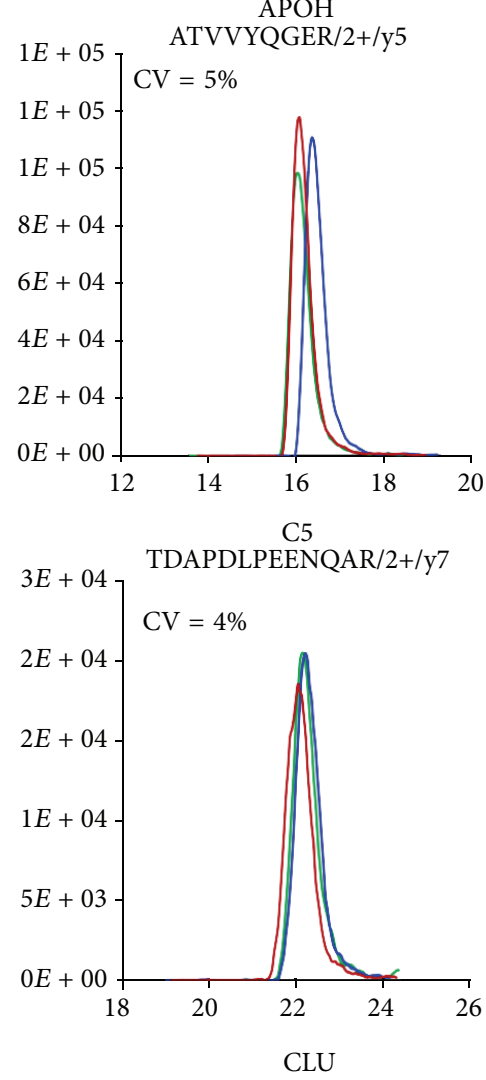

ASSIIDELFQDR/2+/y8

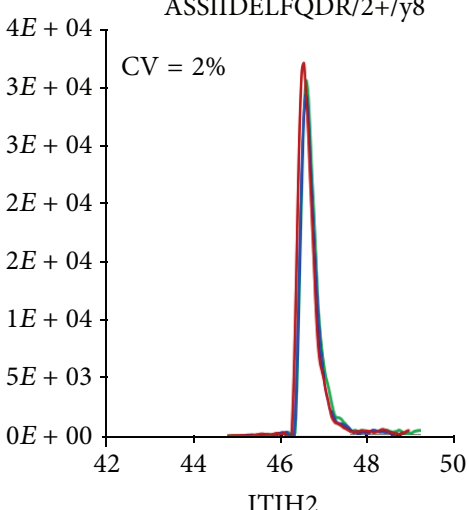

VQSTITSR/2+/y6

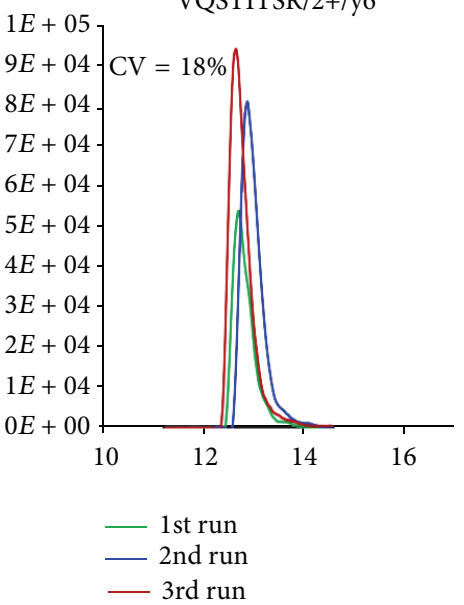

Figure 2: Continued. 

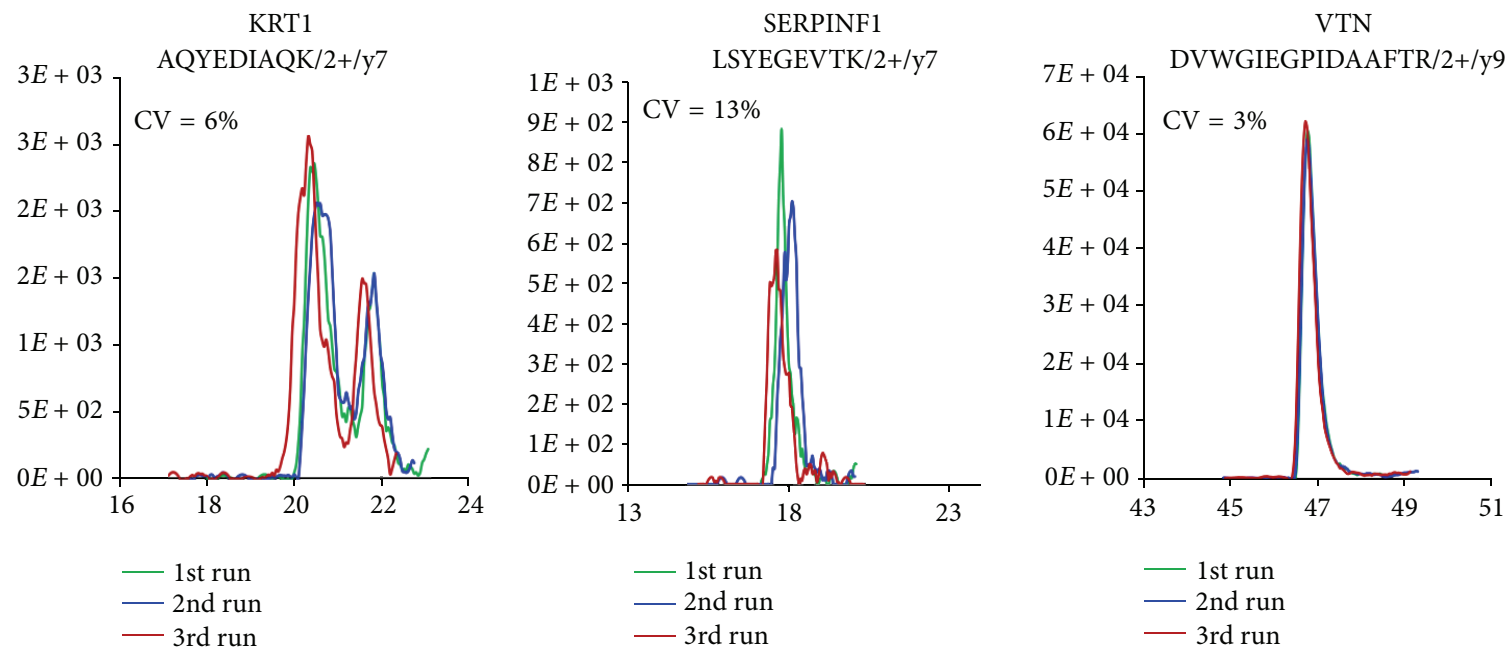

FIGURE 2: The reproducibility of the 3 independent MRM analyses. Before performing the SQ-MRM analysis, we first determined the reproducibility in 3 independent experiments using pooled plasma samples; representative results of 15 proteins are shown. The reproducibility testing showed that APLP2, FN1, ITIH2, and SERPINF1 had higher CV values (19\%, 14\%, 18\%, and 13\%, resp.), while the other proteins had lower CV values.

we should synthesize to obtain the reliable quantitated data. As a preliminary SQ-MRM experiment, we analyzed the correlation of peak area values between two peptides for the 22 proteins with 2 peptides and, then, the peak similarities of two peptides were analyzed by Pearson's correlation analysis. The patterns of peak intensity were highly correlated between two peptides in each protein showing the median value of 0.927 (Supplementary Figure 2). Consequently, the peptide, which had the highest intensity (best detectability) of the multiple peptides per protein, was finally selected and synthesized for SID-MRM analysis ( $>95 \%$ purity).

First, the purity of the SIS heavy peptides for SIDMRM was confirmed by MALDI-TOF analysis (Table 1 and Supplementary Figure 3). All of the SIS heavy peptides had equivalent theoretical and experimental molecular weights. For accurate confirmation of spectral similarity between the experimental spectra obtained in our MRM analysis and the spectra derived from a DR spectral library, we constructed a DR-specific MS/MS spectral library (MS/MS spectra for 3067 peptides); to generate DR-specific MS/MS spectra, a DR sample, which consisted of pooled plasma $(1 \mu \mathrm{g} / \mu \mathrm{L})$ and the 15 SIS peptides mixture $(50 \mathrm{fmol} / \mu \mathrm{L})$, was analyzed by QExactive quadrupole-Orbitrap mass spectrometry (Supplementary Methods).

Prior to performing a second round of MRM verification of the 15 selected candidates, we first checked endogenous light peptide levels by analyzing TEST sample \#1, which was composed of endogenous light peptides (pooled digested plasma, $1 \mu \mathrm{g} / \mu \mathrm{L}$ ) and heavy peptides (50 fmol of the 15 heavy peptides mixture). We were able to confirm low (APLP2, B3GNT1, CD14, and KRT1), middle (C5, C7, FN1, GSN, ITIH2, and SERPINF1), and high (APOA4, APOH, C4B, CLU, and VTN) endogenous concentrations of the proteins, wherein the ranges of low, middle, and high concentrations were defined by comparing endogenous peptide with heavy peptide concentrations $(<10 \mathrm{fmol},<50 \mathrm{fmol}$, and $>50 \mathrm{fmol}$, resp.) (Figure 4). Second, we checked the noise signal of the heavy peptide level by analyzing TEST sample \#2, which was composed of only endogenous light peptides (pooled plasma, $1 \mu \mathrm{g}$ without heavy peptide). We found that the detected signal at the heavy peptide position represented noise when we performed MRM analysis using the individual plasma samples (endogenous light peptide). A range of noise signals were detected for APLP2, B3GNT1, CD14, and GSN, while noise signals were barely detected for the other proteins (Figure 5).

To verify the biomarker candidates using SID-MRM analysis, we evaluated the linearity of the 15 proteins by analyzing a standard curve generated by serial dilutions of a known concentration of a heavy peptide. Next, we determined the LLOD and LLOQ for the 15 selected biomarker candidates. The LLOD was calculated based on the variance of the blank sample and standard deviation (S.D.) of the sample with the lowest level of "spiked" in SIS peptides (see Section 2 for details). Finally, using the heavy peptide mixture as an internal standard, individual plasma concentrations were measured using SID-MRM.

To generate a calibration curve for the 15 proteins, heavy peptides were serially diluted to yield 12 different concentrations (0.1-200 fmol) of the light peptide (digested pooled plasma protein, $1 \mu \mathrm{g}$ ). Each experiment was repeated in triplicate to plot the calibration curve. The area ratio of heavy peptide (12 concentrations from $0.1-200 \mathrm{fmol}$ ) to light peptide (internal standard, $1 \mu \mathrm{g}$ plasma) was determined from the extracted ion chromatogram (XIC) of each selected transition. These calibration curves demonstrated linearity of approximately 3 orders of magnitude for the concentration range, and a strong linear correlation $\left(R^{2}>0.98\right)$ was observed for 13 of the 15 calibration curves, in which APLP2 and B3GNT1 showed lower $R^{2}$ values of 0.96 and 0.94 , respectively (Supplementary Figures $4 \mathrm{~A}$ and $4 \mathrm{~B}$ and Table 2 ). Figure 6 shows the representative calibration curve for the APOA4 peptide (LAPLAEDVR_ $2^{++}$). 


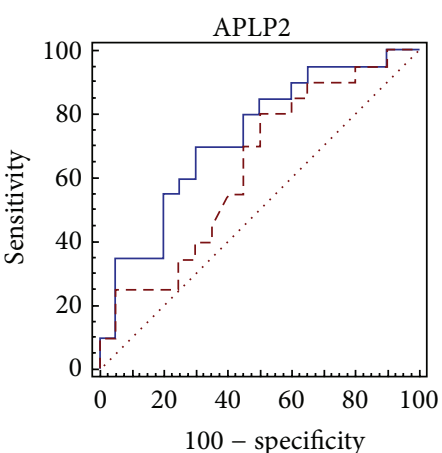

— AUC: 0.72 , No DR versus Mi NPDR - - - AUC: 0.63, No DR versus Mo NPDR

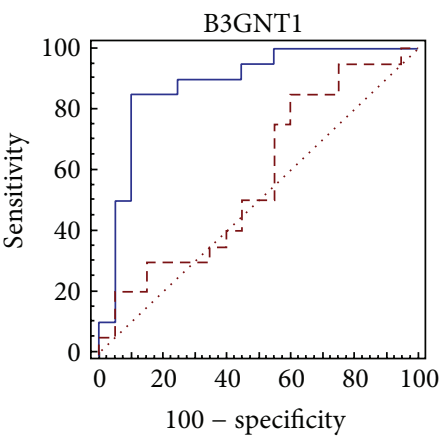

- AUC: 0.88 , No DR versus Mi NPDR - - - AUC: 0.57, No DR versus Mo NPDR

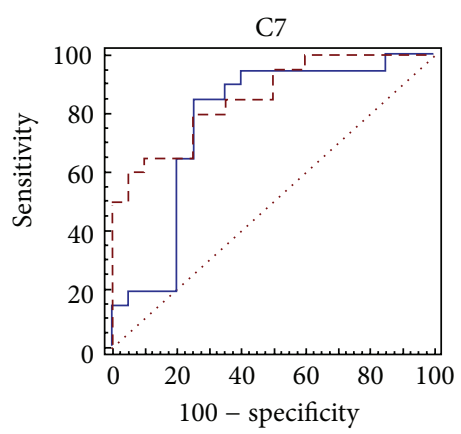

_ AUC: 0.77 , No DR versus Mi NPDR - - - AUC: 0.85, No DR versus Mo NPDR

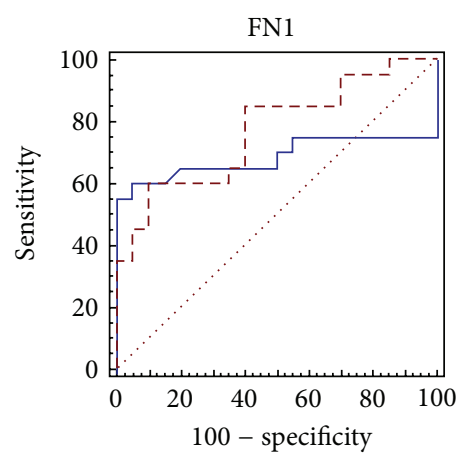

- AUC: 0.68 , No DR versus Mi NPDR

- - - AUC: 0.77, No DR versus Mo NPDR
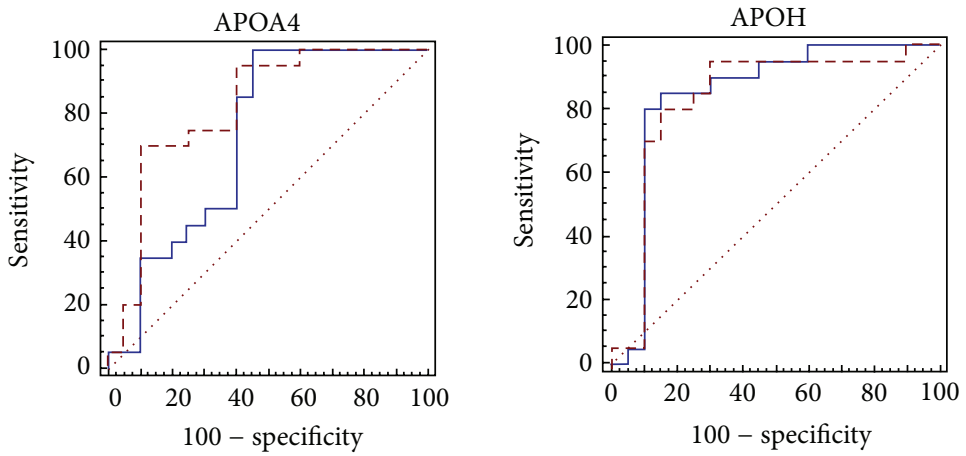

- AUC: 0.72 , No DR versus Mi NPDR

— AUC: 0.84 , No DR versus Mi NPDR - - AUC: 0.82 , No DR versus Mo NPDR - - - AUC: 0.83 , No DR versus Mo NPDR
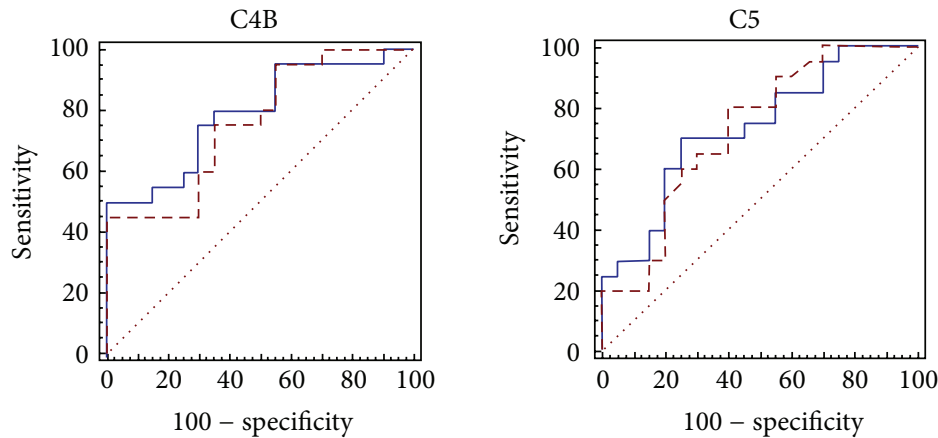

- AUC: 0.79 , No DR versus Mi NPDR _ _ - AUC: 0.76, No DR versus Mo NPDR

- AUC: 0.73 , No DR versus Mi NPDR - - - AUC: 0.72, No DR versus Mo NPDR
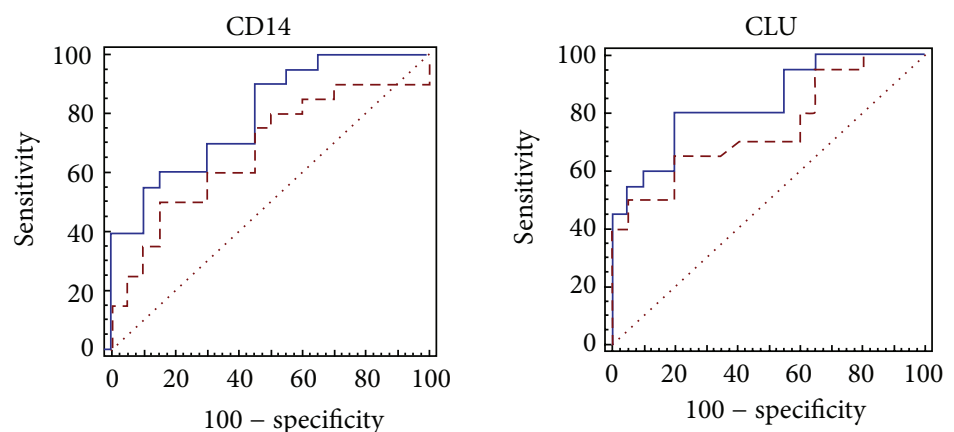

— AUC: 0.79 , No DR versus Mi NPDR _ - - AUC: 0.67, No DR versus Mo NPDR

- AUC: 0.83 , No DR versus Mi NPDR - _ - AUC: 0.74 , No DR versus Mo NPDR
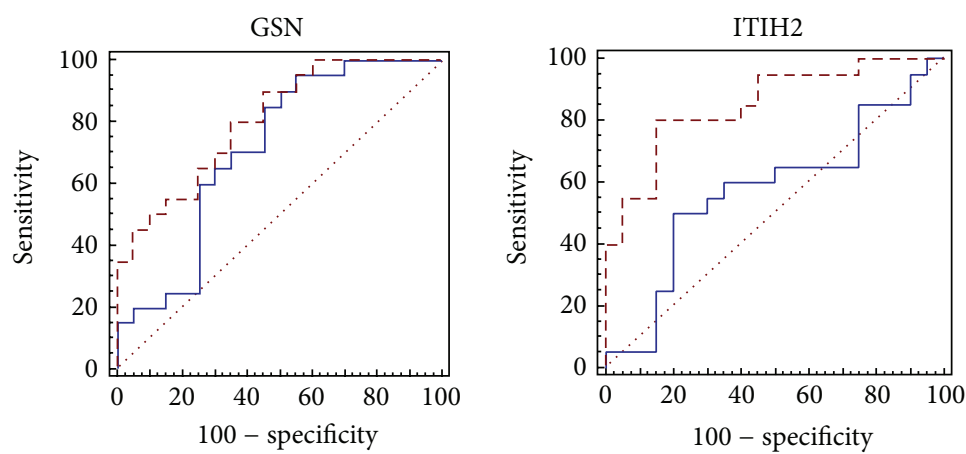

AUC: 0.57 , No DR versus Mi NPDR - - - AUC: 0.85 , No DR versus Mo NPDR

Figure 3: Continued. 

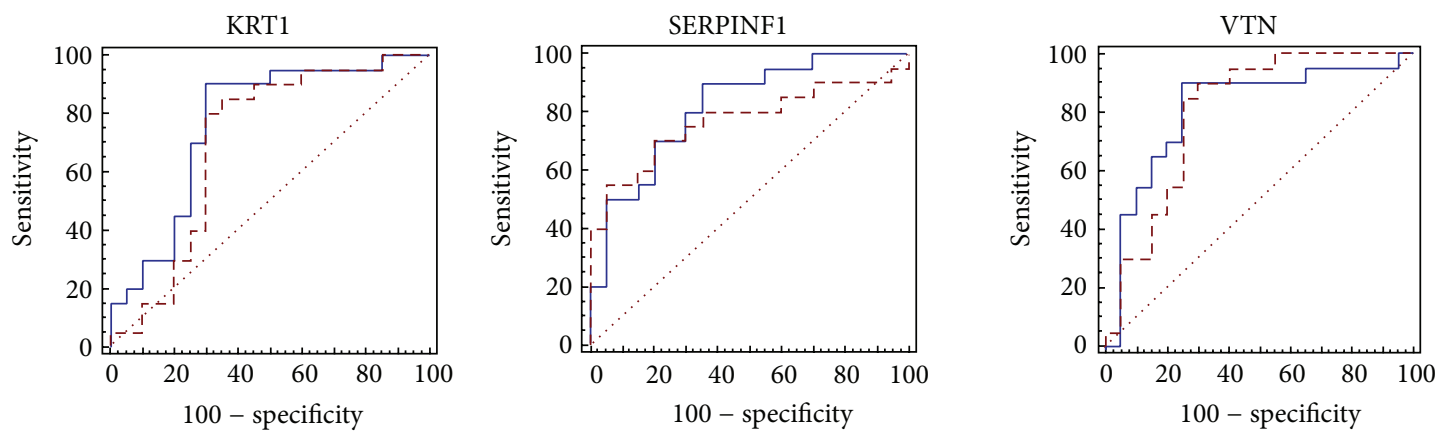

_ AUC: 0.76 , No DR versus Mi NPDR

_ AUC: 0.82 , No DR versus Mi NPDR
_ _ - AUC: 0.77 , No DR versus Mo NPDR

— AUC: 0.82 , No DR versus Mi NPDR

- - AUC: 0.81, No DR versus Mo NPDR

FIGURE 3: Verification of biomarker candidates using SQ-MRM analysis. To verify the selected biomarker candidates, we measured the relative concentrations of the 39 candidates in the samples from the No DR, Mi NPDR, and Mo NPDR groups. In the SQ-MRM analysis, we confirmed a differential measurement of these proteins among the No DR $(n=20)$, Mi NPDR $(n=20)$, and Mo NPDR $(n=20)$ groups. ROC with AUC values were generated based on the SQ-MRM analysis. In the SQ-MRM analysis, we "spiked" the samples with $50 \mathrm{fmol}$ betaGAL (GDFQFNISR, $542.3 / 636.3 \mathrm{~m} / z$ ).

To determine the concentration range of quantification for each peptide, we determined the LLOD and LLOQ for each of the 15 proteins. The LLOD and LLOQ for 4 proteins (APOA4, CLU, ITIH2, and VTN) were very low (0.06$0.32 \mathrm{fmol} / \mu \mathrm{L}$ and $0.19-0.96 \mathrm{fmol} / \mu \mathrm{L}$, resp.), among which CLU had the lowest LLOD and LLOQ $(0.06 \mathrm{fmol} / \mu \mathrm{L}$ and $0.09 \mathrm{fmol} / \mu \mathrm{L}$, resp.). By contrast, the LLOD and LLOQ ranges for APLP2, B3GNT1, CD14, and GSN were higher (2.57$5.96 \mathrm{fmol} / \mu \mathrm{L}$ and $7.71-17.88 \mathrm{fmol} / \mu \mathrm{L}$, resp.), and CD14 had the highest LLOD and LLOQ $(5.96 \mathrm{fmol} / \mu \mathrm{L}$ and $17.88 \mathrm{fmol} / \mu \mathrm{L}$, resp.) (Table 1 and Supplementary Figures $4 \mathrm{~A}$ and $4 \mathrm{~B}$ ).

Using the heavy peptide mixture $(50 \mathrm{fmol} / \mu \mathrm{L})$ of the 15 proteins as an internal standard, individual plasma samples were validated using SID-MRM analysis. In this analysis of individual samples, we could confirm a differential concentration of these proteins among the No DR $(n=20)$, Mi NPDR $(n=20)$, and Mo NPDR $(n=20)$ groups. Figure 7 shows a representative result of the individual SID-MRM analysis for the ITIH2_peptide $\left(\right.$ VQSTITSR $\left._{2}{ }^{++}\right)$, depicting a differential concentration range in 60 individual plasma samples, the average elution time for 15 peptides, an XIC for heavy and light peptides of a peptide (VQSTITSR_2 ${ }^{++}$), and a MS/MS spectrum for a fragmented peptide (VQSTITSR_2 ${ }^{++}$). In our individual SID-MRM analysis, the CV\% for retention time (RT) in the No DR, Mi NPDR, and Mo NPDR groups showed ranges of $0.87-8.35,0.23-4.81$, and 0.11-4.92 min, respectively (Table 1 and Supplementary Figure 5).

In this quantitative analysis (Figures $8(\mathrm{a})$ and $8(\mathrm{~b})$ ), the endogenous concentrations of 12 proteins (APOA4, APOH, B3GNT1, C4B, C5, C7, CD14, CLU, GSN, ITIH2, KRT1, and VTN) were significantly different between the No DR and Mi NPDR (AUC value > 0.7) and the No DR and Mo NPDR (AUC value $>0.7$ ) groups. By contrast, no significant difference in the concentrations of 3 proteins (APLP2, FN1, and SERPINF1) was observed between the No DR and NPDR groups.
3.7. Construction of a Multimarker Panel Based on the SIDMRM Results. Statistical analyses was carried out using the SID-MRM data for the 15 proteins, which were obtained from patients with No DR $(n=20)$, Mi NPDR $(n=20)$, and Mo NPDR $(n=20)$. First, we chose a multimarker panel that showed the best discriminatory power between the No DR and Mo NPDR groups. We then applied the multimarker panel to evaluate its discriminatory power between the No DR and Mi + Mo NPDR groups. Because Mi NPDR represents an early stage of NPDR, it was not easy to observe differences between the No DR and Mi NPDR groups. To screen NPDR, it may be more reliable to screen Mo NPDR than Mi NPDR. Therefore, to determine the performance of the multimarker panel, we compared the No DR and Mo NPDR samples to measure its classification power.

Student's $t$-test was performed to compare the No DR and Mo NPDR groups, and 11 proteins showed significant differences (APOA4, APOH, B3GNT1, C4B, C5, C7, CD14, CLU, GSN, ITIH2, and KRT1). Before constructing the multimarker panel, we conducted a correlation analysis between all of the markers to test for multicollinearity among the 11 candidates that we verified by SID-MRM analysis using linear regression analysis. Multicollinearity generally indicates that there is a high correlation of at least one independent variable with a combination of the other independent variables. The variation inflation factor (VIF) and tolerance of the 11 candidate ranged from 1.5 to 7.2 and 0.13 to 0.65 , respectively (Supplementary Figure 6A). Generally, multicollinearity becomes problematic when the VIF value is greater than 10 and the tolerance is close to 0 .

To construct prediction models using the variables that contribute to the discrimination power, we performed stepwise MANOVA to determine whether each candidate should be included in a multimarker panel. The MANOVA test not only could reveal the significant differences between 2 groups but also showed relationships between 2 dependent variables and independent or dependent variables. From the stepwise MANOVA (Supplementary Figure 6B), 4 verified markers 


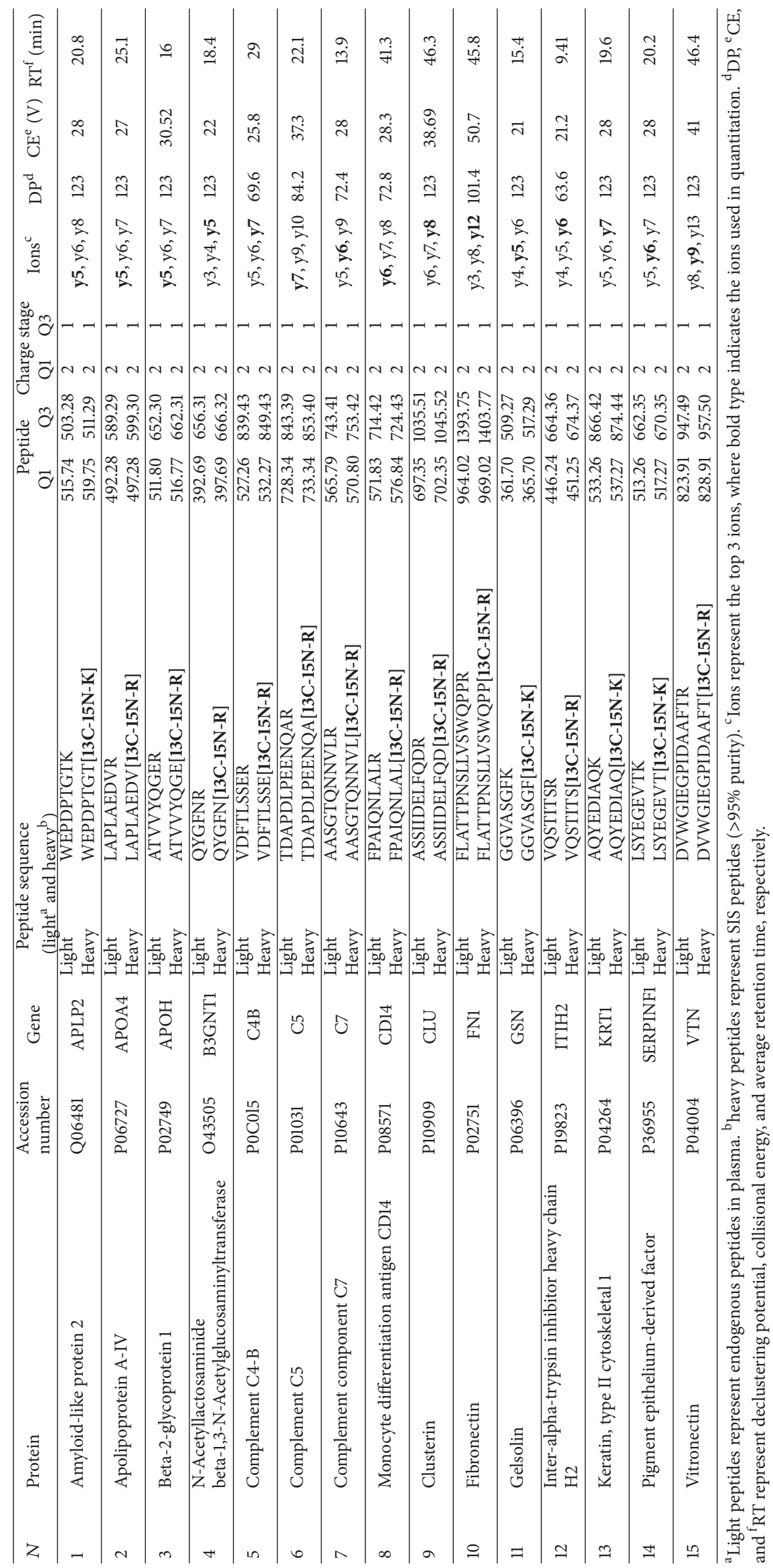



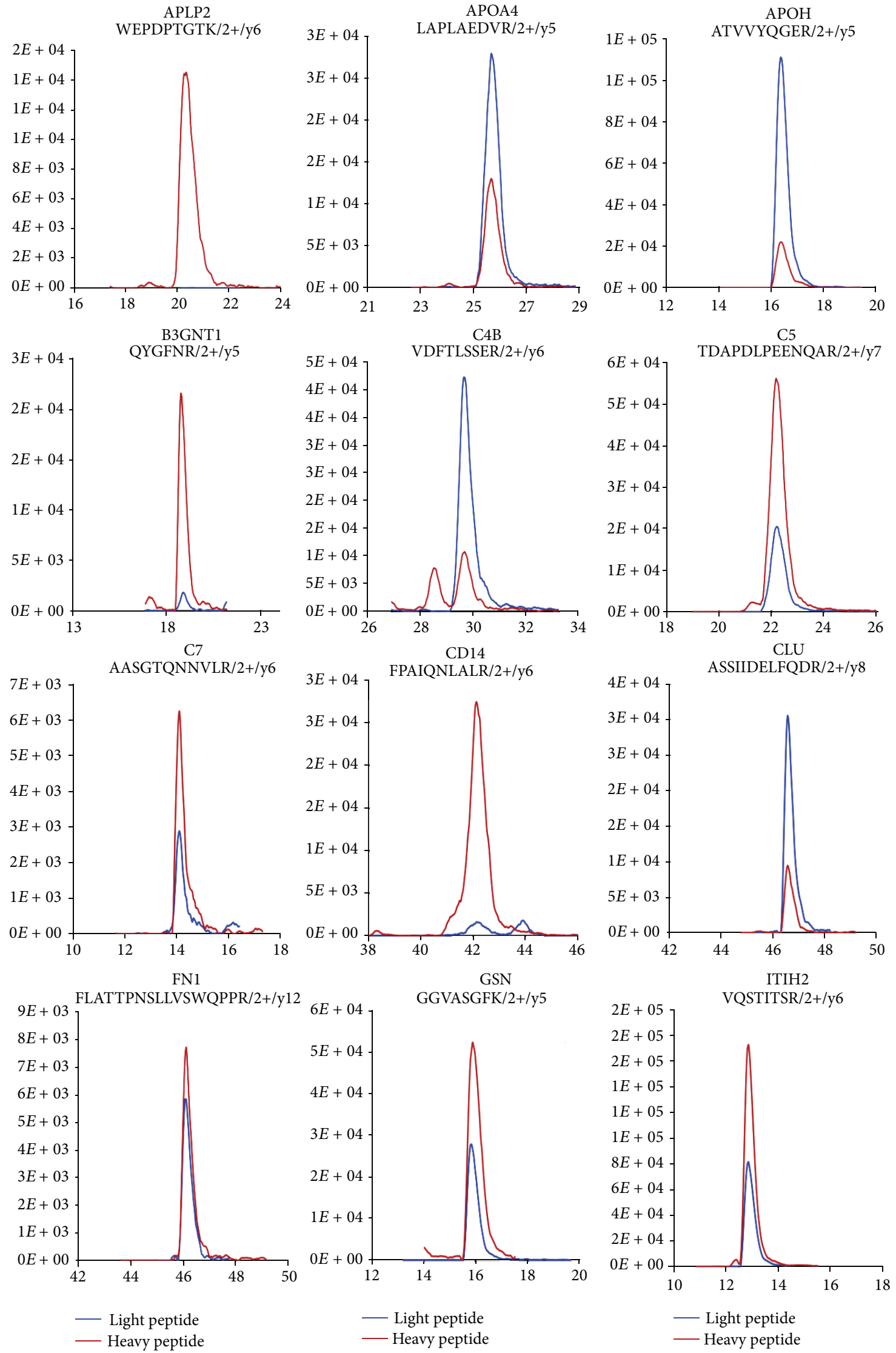

Figure 4: Continued. 

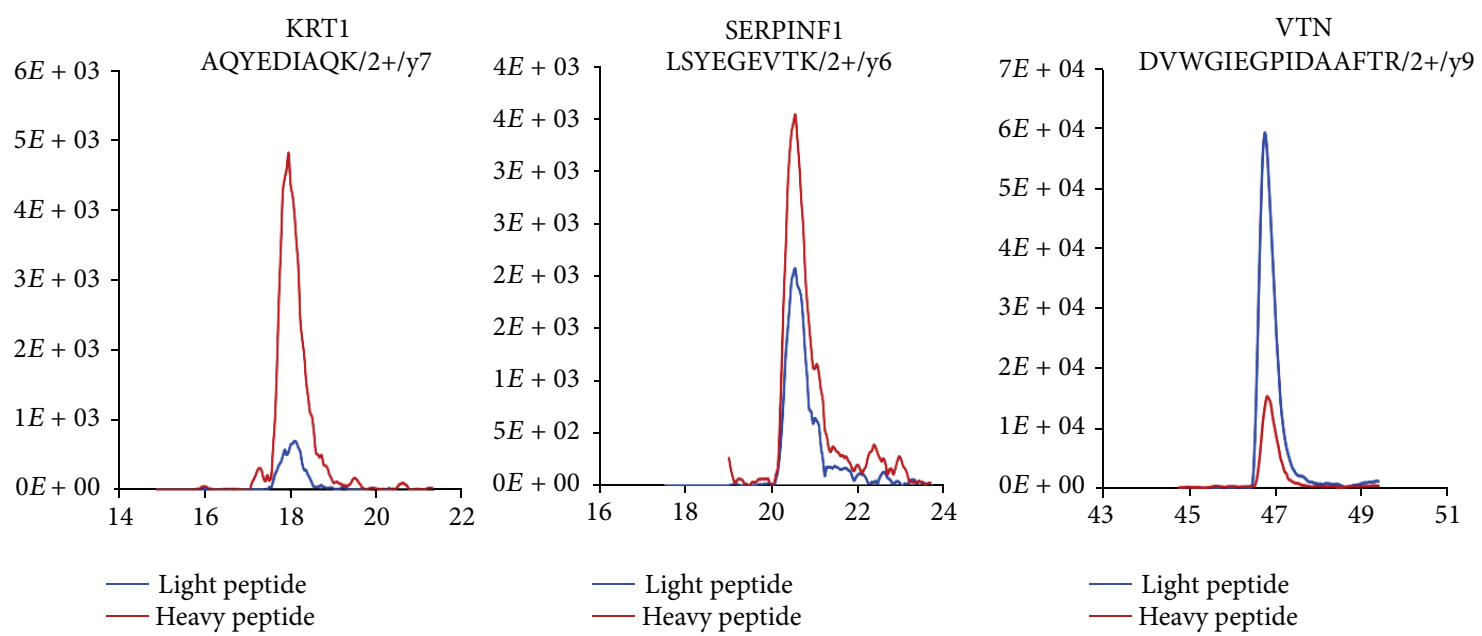

FIGURE 4: Detection of endogenous light peptide levels in pooled plasma. The endogenous light peptide levels in pooled plasma were measured by analyzing TEST sample \#1, which was composed of endogenous light peptides (pooled digested plasma, $1 \mu \mathrm{g} / \mu \mathrm{L}$ ) and heavy peptides ( $50 \mathrm{fmol}$ of the heavy peptide mixture). From this analysis, low (APLP2, B3GNT1, CD14, and KRT1), middle (C5, C7, FN1, GSN, ITIH2, and SERPINF1), and high (APOA4, APOH, C4B, CLU, and VTN) concentration ranges of proteins were confirmed.

(ITIH2, APOA4, C7, and CLU) were selected for inclusion in a multimarker panel.

3.8. Evaluation of a 4-Protein Multimarker Panel in the No DR versus Mo NPDR Comparison. The 4 verified markers were used to construct a 4-marker panel, which was evaluated for its discriminatory performance. An algorithm of LDA was used to examine the discriminatory power in the No DR versus Mo NPDR comparison; subsequently, LOOCV was used to avoid overfitting. In the LDA model of the 4marker panel, 19 of 20 patients in both the No DR and the Mo NPDR groups were correctly classified with an error rate of $5 \%$ (Figure 9(b)). To compare the discriminatory power of the 4-marker panel with the single best marker (ITIH2), a model coupled with LDA for the single best marker (ITIH2) was also built in which LOOCV was performed in the No DR versus Mo NPDR comparison (Figures 9(a) and 9(b)). As indicated by the SID-MRM results (Figure 8(a)), ITIH2 showed the highest AUC value among the 15 verified candidate proteins and was therefore selected as the best single marker.

In the LDA model, for the parameters of sensitivity (0.95), specificity (0.95), error rate (5\%), and AUC value (0.99), the 4-marker panel was superior to the best single marker (ITIH2) (Figure 9(b)). The best single marker model showed a lower sensitivity (0.85), specificity (0.85), AUC value (0.91), and a higher error rate (15\%) when compared to the 4 -marker panel.

3.9. Evaluation of the 4-Marker Panel in the No DR versus Mi+ Mo NPDR Comparison. Next, we used the 4-marker panel to evaluate its discriminatory power in the No DR versus $\mathrm{Mi}+$ Mo NPDR comparison. In the LDA model, the 4-marker panel showed that 19 of 20 No DR patients and 36 of $40 \mathrm{Mi}+$ Mo NPDR patients were correctly classified, while the best single marker showed that 13 of 20 No DR patients and 22 of $40 \mathrm{Mi}+\mathrm{Mo}$ NPDR patients were correctly classified. The 4-marker panel (sensitivity, 0.90; specificity, 0.95; error rate,
8\%; and AUC, 0.93) showed better performance than the best single marker (sensitivity, 0.55; specificity, 0.65; error rate, 41\%; and AUC, 0.67) (Figures 9(a) and 9(b)).

\section{Discussion}

4.1. Selection of DR Biomarker Candidates from the First MRM Analysis. To select biomarker candidates, we performed data-mining on previously published DR-related studies and our experimental data. We included human, mouse, and rat datasets and selected 1202 proteins (data not shown).

However, considering the low number of common proteins between human vitreous fluid and the retina of animal models, along with the use of human plasma in the biomarker validation step, we decided to only use the human datamining data sets for the selection of biomarker candidates. From the human data-mining analysis, a total of 490 proteins were identified. To confirm the MRM transition, 96 proteins (387 peptides and 1870 transitions) were ultimately selected.

Based on a MASCOT search to confirm each transition in pooled plasma, 39 proteins were selected, including 22 proteins that had 2 peptides and 17 proteins that had 1 peptide (Supplementary Table 6). Briefly, 1870 transitions (387 peptides) for 96 proteins that were found in pooled plasma were selected. Based on the MASCOT search results, 296 transitions (61 peptides) of 1870 transitions (387 peptides) were finally identified as peptides derived from the original protein. The data indicated that $319(82.4 \%)$ of 387 peptides were coeluted with other peptides, while $61(15.8 \%)$ of 387 peptides were true peptides derived from the original proteins, which is a very low recovery efficiency. According to Addona et al. [34], the most common sample-related source of quantification errors in MRM is ion interference from other coeluting peptides.

The 39 candidates were then examined in the SQ-MRM quantification analysis, and we found 15 proteins that were 
APLP2

WEPDPTGTK/2+/y5

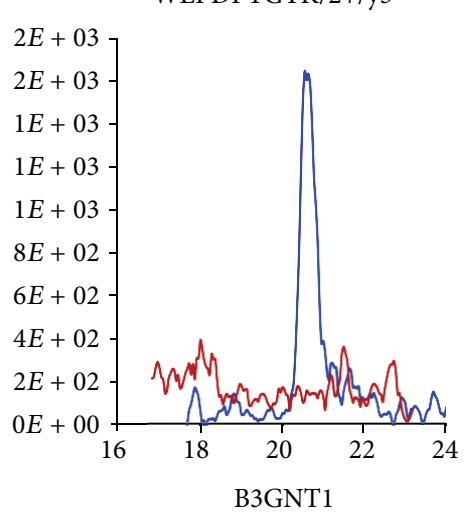

QYGFNR/2+/y5

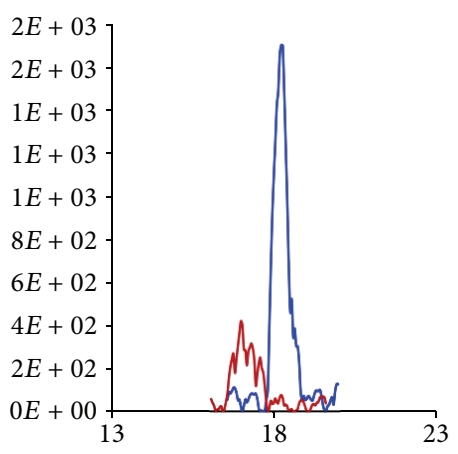

C7

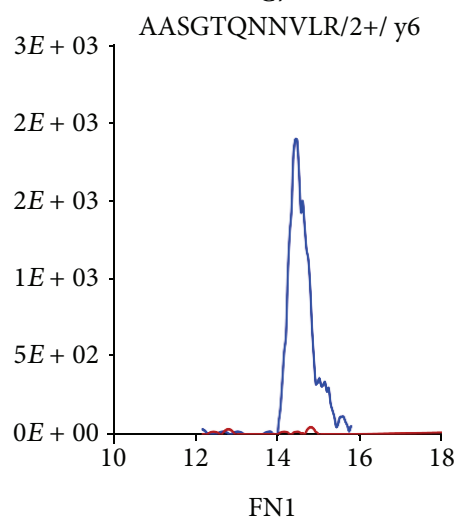

FLATTPNSLLVSWQPPR/2+/y12
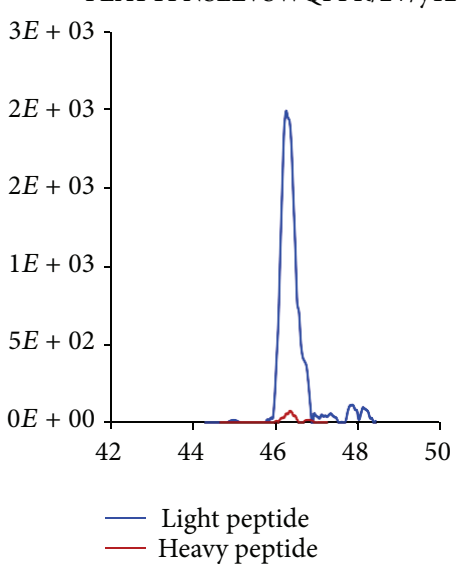

APOA4

LAPLAEDVR/2+/y5

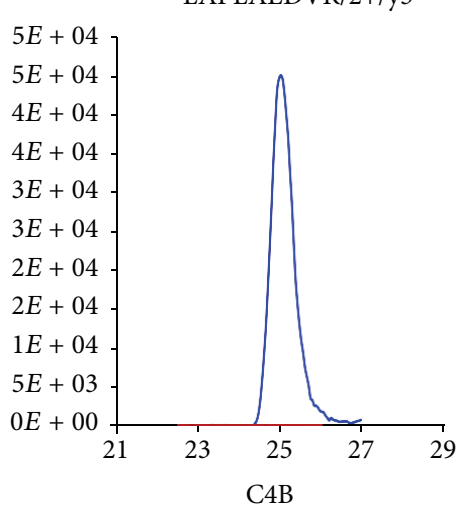

VDFTLSSER/2+/y7

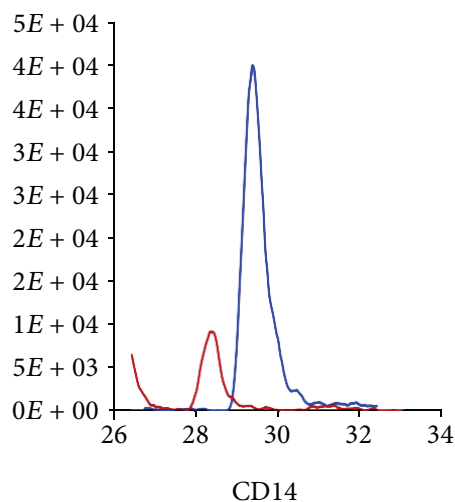

FPAIQNLALR/2+/y6

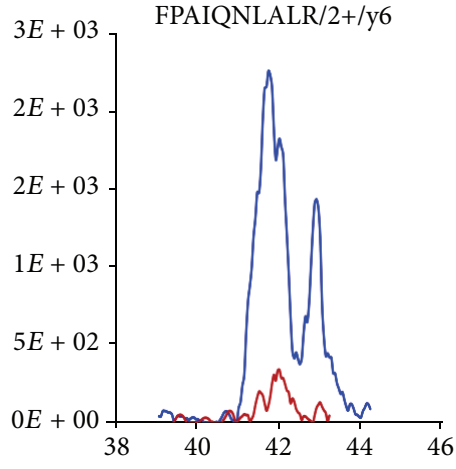

GSN

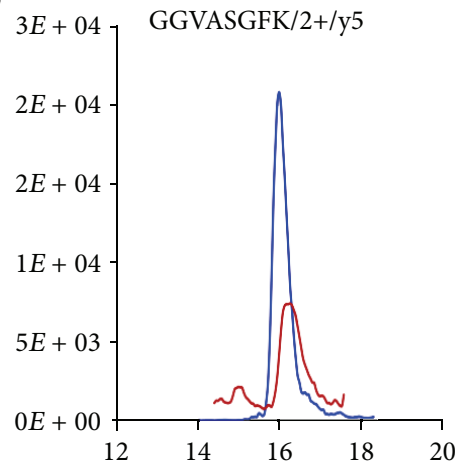

— Light peptide
— Heavy peptide

FIGURE 5: Continued.
$\mathrm{APOH}$

ATVVYQGER/2+/y5

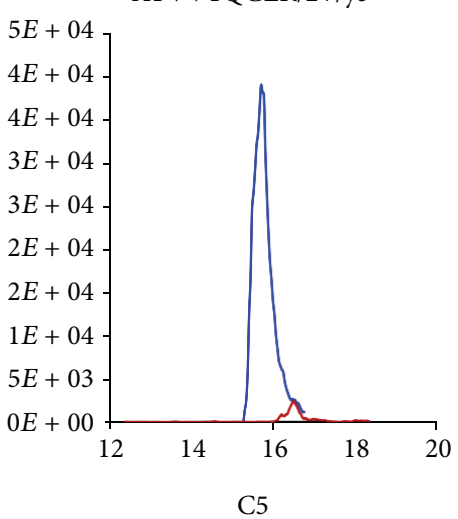

TDAPDLPEENQAR/2+/y7

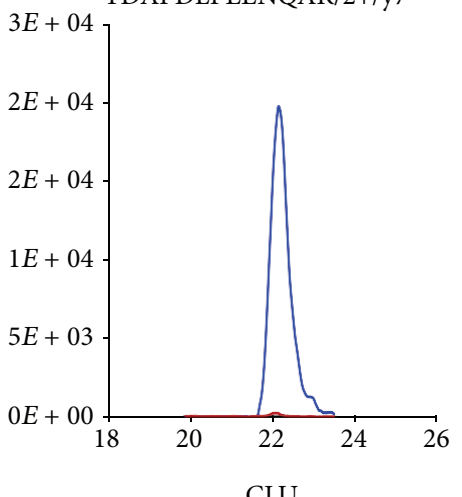

ASSIIDELFQDR/2+/y8

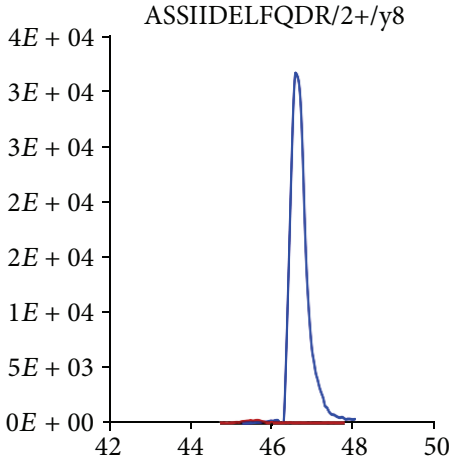

ITIH2

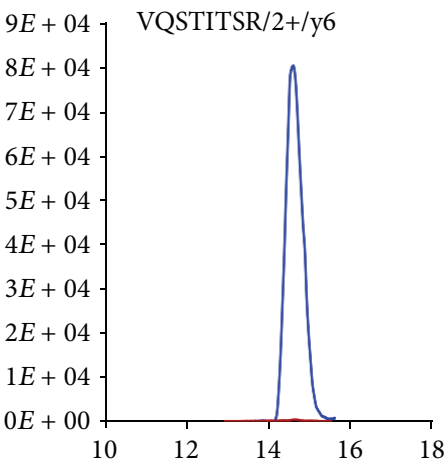

- Light peptide - Heavy peptide 

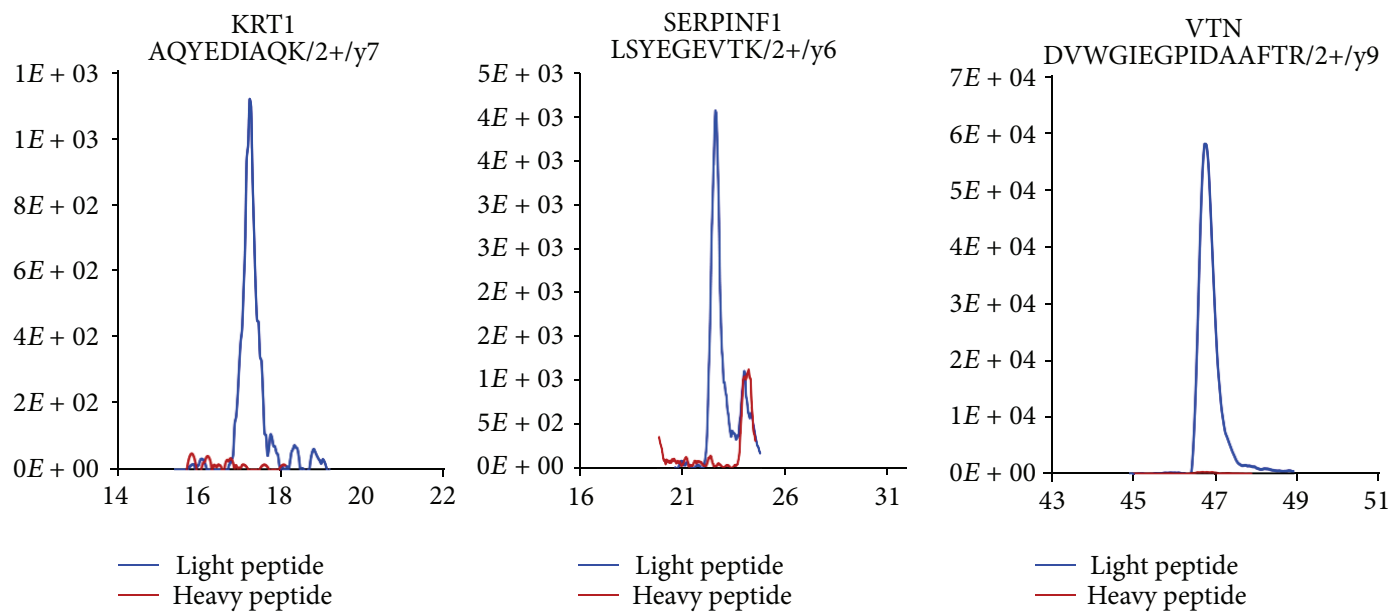

Figure 5: The detection of noise signals for heavy peptides in the pooled plasma. Noise signals of heavy peptides were assessed by analyzing TEST sample \#2, which was composed of only endogenous light peptides (pooled plasma, $1 \mu \mathrm{g}$ without heavy peptide). A range of noise signals was detected for APLP2, B3GNT1, CD14, and GSN, while a noise signal was barely detected for the other proteins.

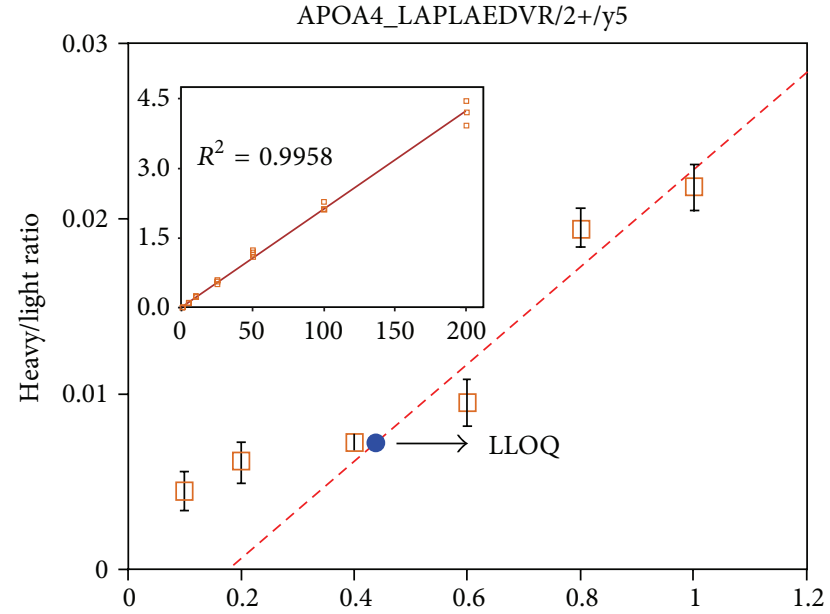

FIGURE 6: Representative calibration curves. To generate calibration curves for the 15 proteins, heavy peptides were serially diluted (12 concentrations, $0.1-200 \mathrm{fmol}$ ) with the light peptide as an internal standard (digested pooled plasma protein, $1 \mu \mathrm{g}$ ) that was added into each serially diluted sample. A representative calibration curve for APOA4_LAPLAEDVR $\left(\mathrm{y}^{++}\right)$is shown. Insets show the linear ranges of the curves using $R^{2} \geq 0.99$ to determine the LLOQ.

significantly differentially expressed between the No DR, Mi NPDR, and Mo NPDR groups.

4.2. A Comparison of the Mean Concentrations in the No $D R, M i N P D R$, and Mo NPDR Groups. To measure the concentration of the 15 proteins in the No DR $(n=20)$, Mi NPDR $(n=20)$, and Mo NPDR $(n=20)$ groups, we first determined the LLOQ range using the pooled plasma samples. For the 4 proteins (APOA4, CLU, ITIH2, and VTN) that had a very low LLOQ range $(0.19-0.96 \mathrm{fmol} / \mu \mathrm{L})$, all of the measured average concentrations had a higher LLOQ range in the No DR, Mi NPDR, and Mo NPDR groups
(Table 2 and Supplementary Figures 4B and 7). To compare the concentration ranges between the normal and NPDR groups, we identified the normal plasma concentrations reported in the literature $[9,35-38]$. The previously reported concentrations of APOA4 [35] and ITIH2 [36] were higher or similar when compared to the No DR, Mi NPDR, and Mo NPDR groups, while the reported concentrations of CLU $[35,36]$ and VTN $[35,36]$ were lower than in the DR groups reported here (Table 2 and Supplementary Figure 7).

By contrast, APLP2, B3GNT1, CD14, and GSN had a higher LLOQ range (7.71-17.88 fmol $/ \mu \mathrm{L})$. The average concentrations of CD14 and APLP2 in the No DR, Mi NPDR, and Mo NPDR groups were higher than the LLOQ concentration range, whereas the average concentrations of B3GNT1 and GSN were lower than the LLOQ concentration range. For APLP2 and B3GNT1, we assumed that the high LLOD levels resulted from low endogenous light peptide concentrations and high levels of noise signals. The concentrations of CD14 [38] and GSN [36] in normal plasma were higher than the average concentrations in the No DR, Mi NPDR, and Mo NPDR groups (Table 2 and Supplementary Figure 7). However, there were no values available in the literature for the normal plasma concentration ranges of APLP2 and B3GNT1, so we could not make a comparison between the reported and measured concentrations.

Among the 15 selected proteins, the other $7(\mathrm{APOH}$, C4B, C5, C7, FN1, KRT1, and SERPINF1) had a midLLOQ range $(1.01-3.93 \mathrm{fmol} / \mu \mathrm{L})$ in this study. The average measured concentrations for all 7 proteins in the No DR, Mi NPDR, and Mo NPDR groups were higher than the LLOQ range (Table 2 and Supplementary Figure 7). The previously reported concentration ranges of $\mathrm{C} 7[9,36]$ and FN1 [9, 35-37] were higher in normal plasma, whereas those of C4B [36] and SERPINF1 [36] were lower than the average concentration in the No DR, Mi NPDR, and Mo NPDR groups (Table 2 and Supplementary Figure 7). The previously published concentration of APOH [36] in normal plasma was similar to the measured average concentrations 


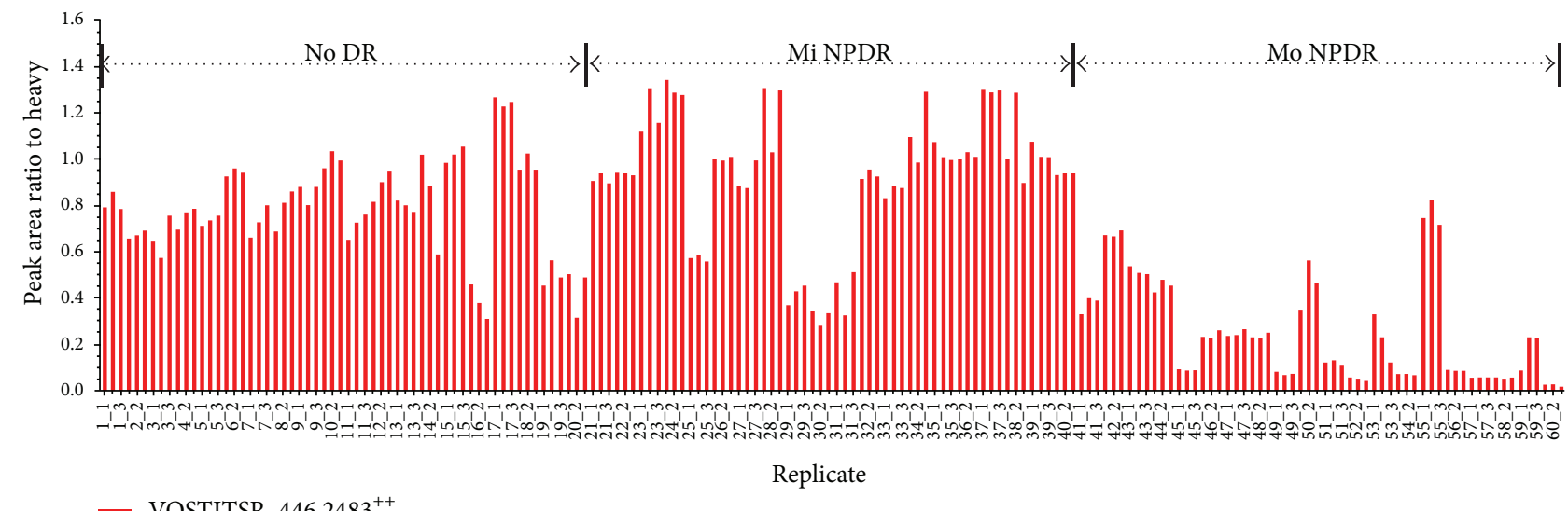

(a)

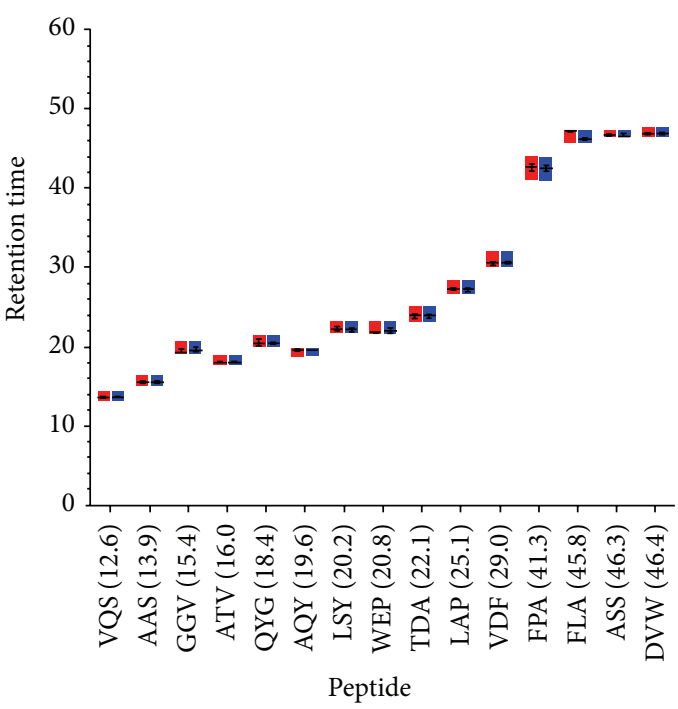

(b)

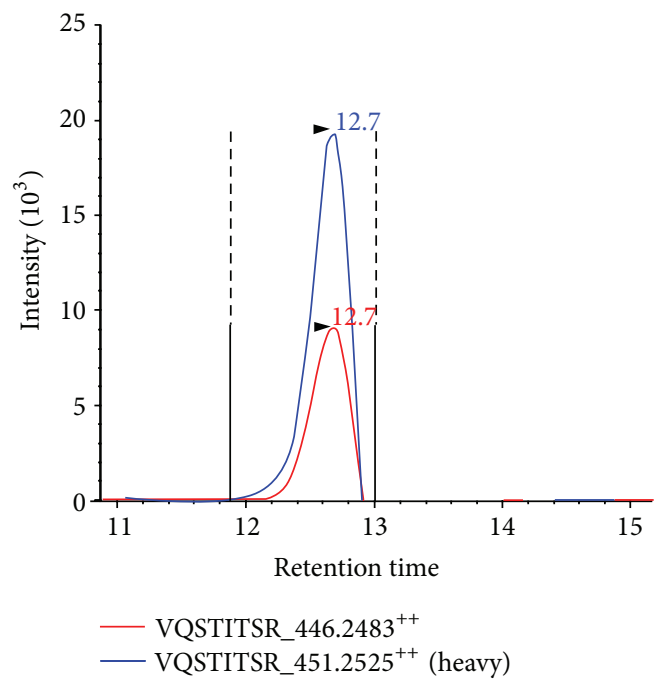

(c)

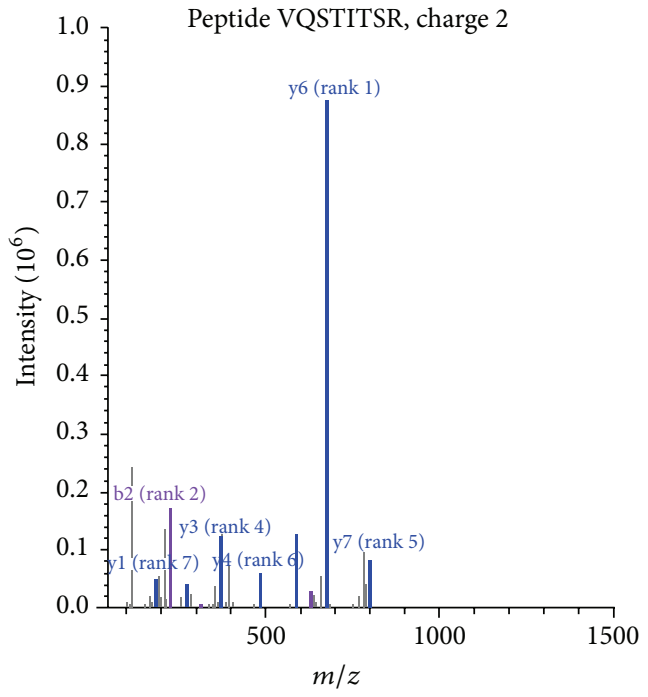

(d)

FIGURE 7: Representative MRM results of the ITIH2 analysis in the No DR, Mi NPDR, and Mo NPDR groups. A representative MRM result for ITIH2-peptide (VQSTITSR_2 $2^{++}$) is shown. The differential concentration range in 60 individual plasmas (a), elution time points for each peptide (b), XIC for the heavy and light peptides of VQSTITSR_2 $2^{++}$(c), and the MS/MS spectrum for fragmented peptides (d) are indicated. In our individual SID-MRM analysis, the CV\% for retention time (RT) in the No DR, Mi NPDR, and Mo NPDR groups showed ranges of $0.87-8.35,0.23-4.81$, and $0.11-4.92$, respectively. All images were extracted using the Skyline program. 
APLP2

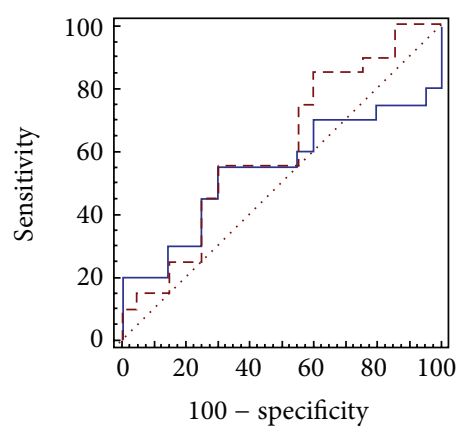

— AUC: 0.54 , No DR versus Mi NPDR

- - - AUC: 0.61, No DR versus Mo NPDR

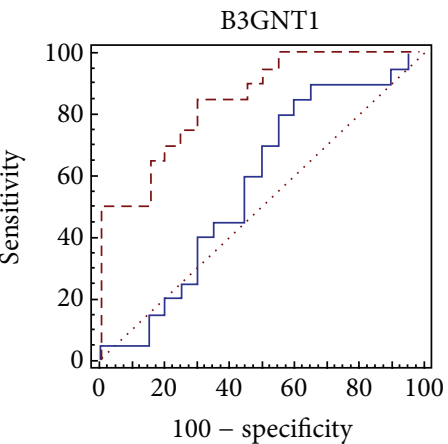

- AUC: 0.57 , No DR versus Mi NPDR

- - - AUC: 0.85, No DR versus Mo NPDR

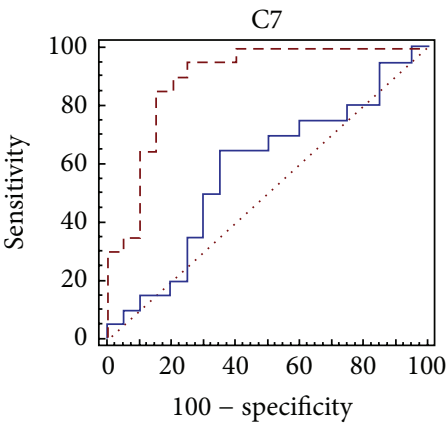

— AUC: 0.58 , No DR versus Mi NPDR

- - - AUC: 0.89, No DR versus Mo NPDR

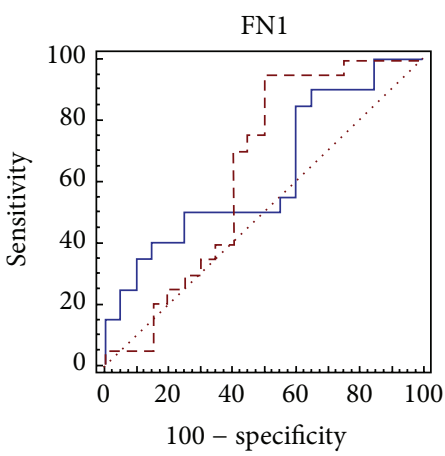

AUC: 0.62 , No DR versus Mi NPDR

- - - AUC: 0.64, No DR versus Mo NPDR
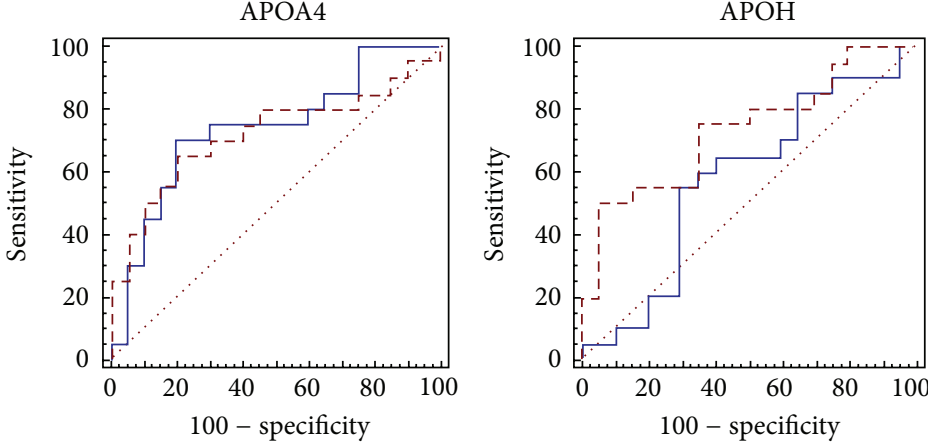

— AUC: 0.73 , No DR versus Mi NPDR

AUC: 0.72 , No DR versus Mo NPDR

— AUC: 0.57 , No DR versus Mi NPDR

_ _ AUC: 0.73, No DR versus Mo NPDR
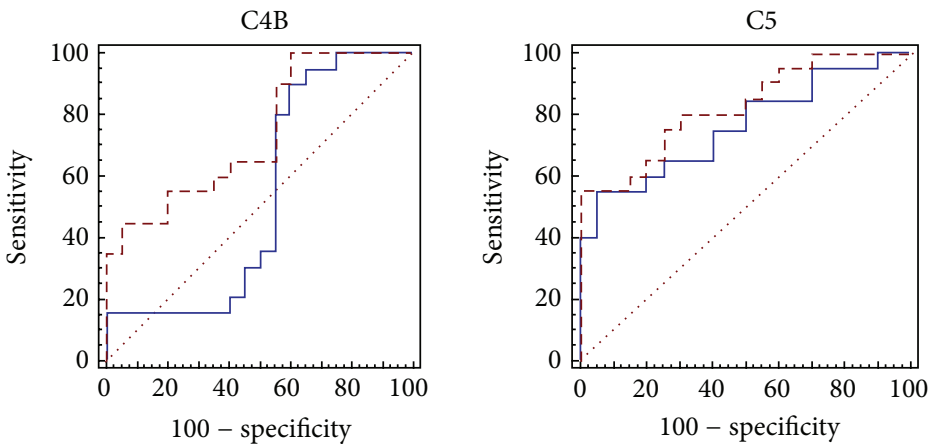

- AUC: 0.53 , No DR versus Mi NPDR

AUC: 0.76 , No DR versus Mi NPDR - - AUC: 0.74, No DR versus Mo NPDR

- - - AUC: 0.82, No DR versus Mo NPDR
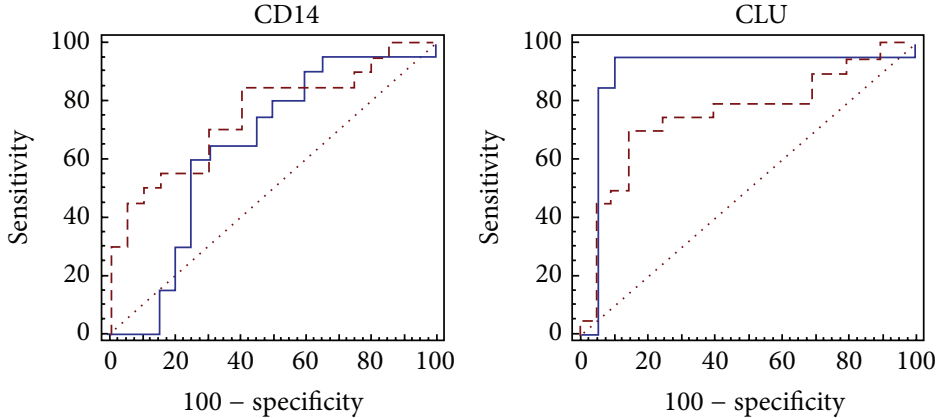

— AUC: 0.64 , No DR versus Mi NPDR

- AUC: 0.89 , No DR versus Mi NPDR - - - AUC: 0.75, No DR versus Mo NPDR
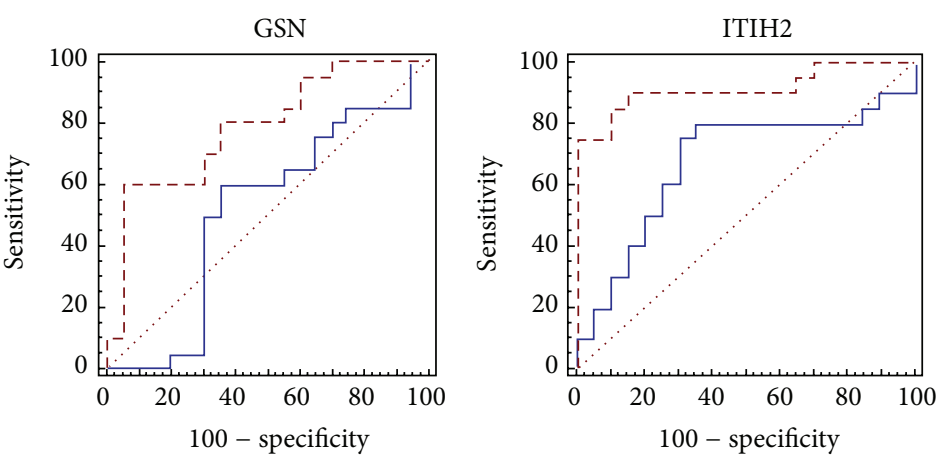

- AUC: 0.69, No DR versus Mi NPDR AUC: 0.67, No DR versus Mi NPDR - - - AUC: 0.72, No DR versus Mo NPDR

Figure 8: Continued. 

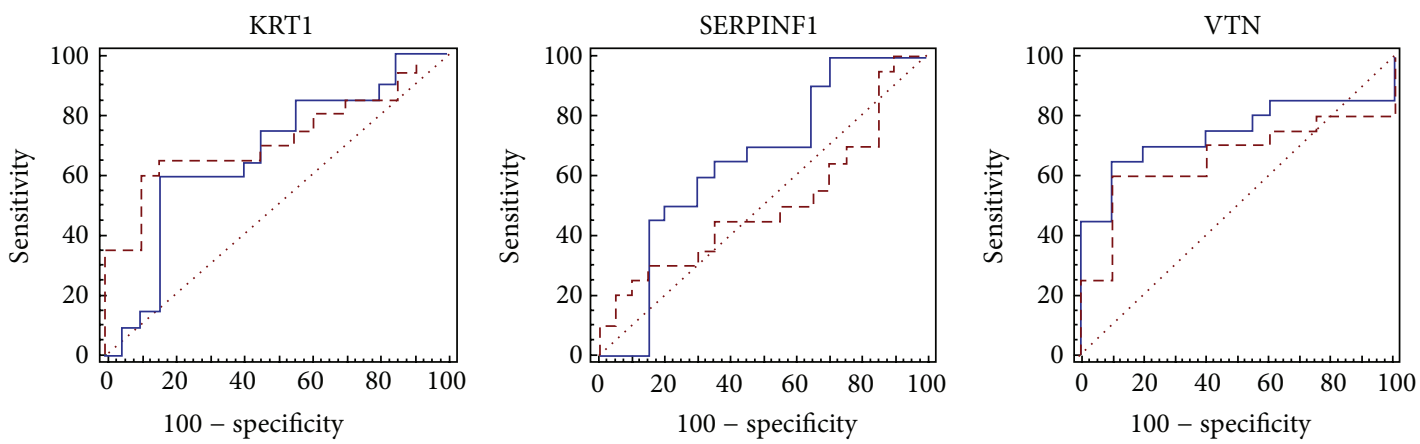

— AUC: 0.67 , No DR versus Mi NPDR

- AUC: 0.65 , No DR versus Mi NPDR

- AUC: 0.74 , No DR versus Mi NPDR

- - - AUC: 0.72 , No DR versus Mo NPDR

- - - AUC: 0.50, No DR versus Mo NPDR

- - - AUC: 0.65 , No DR versus Mo NPDR

(a)
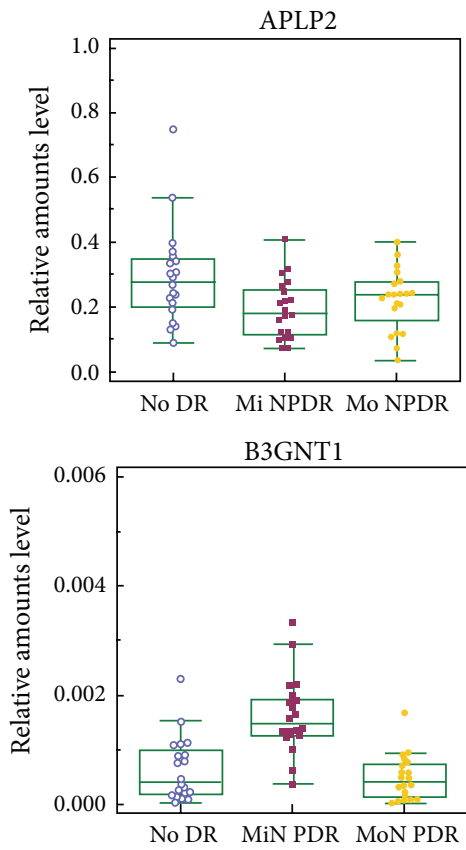

C7

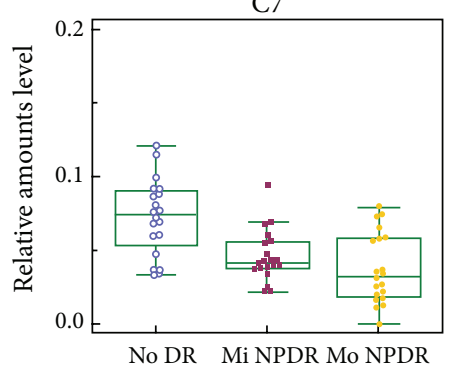

FN1

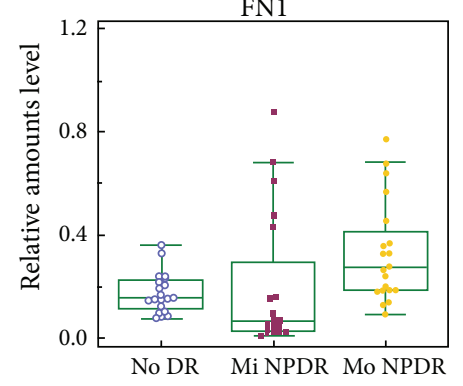

APOA4

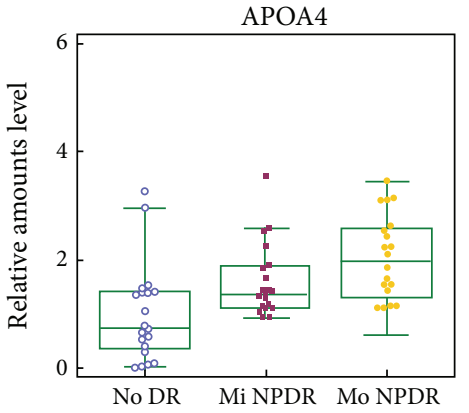

C4B
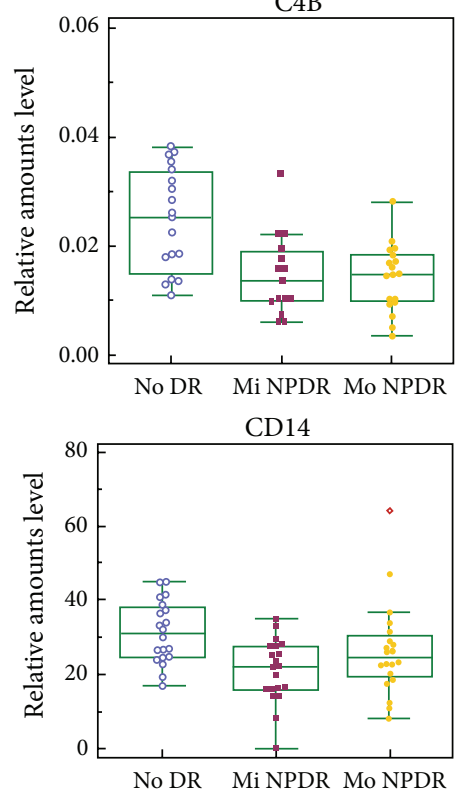

GSN

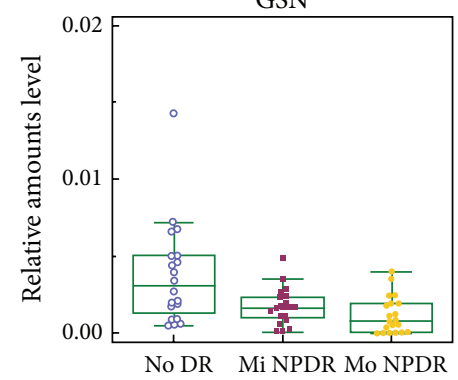

FIgURE 8: Continued.
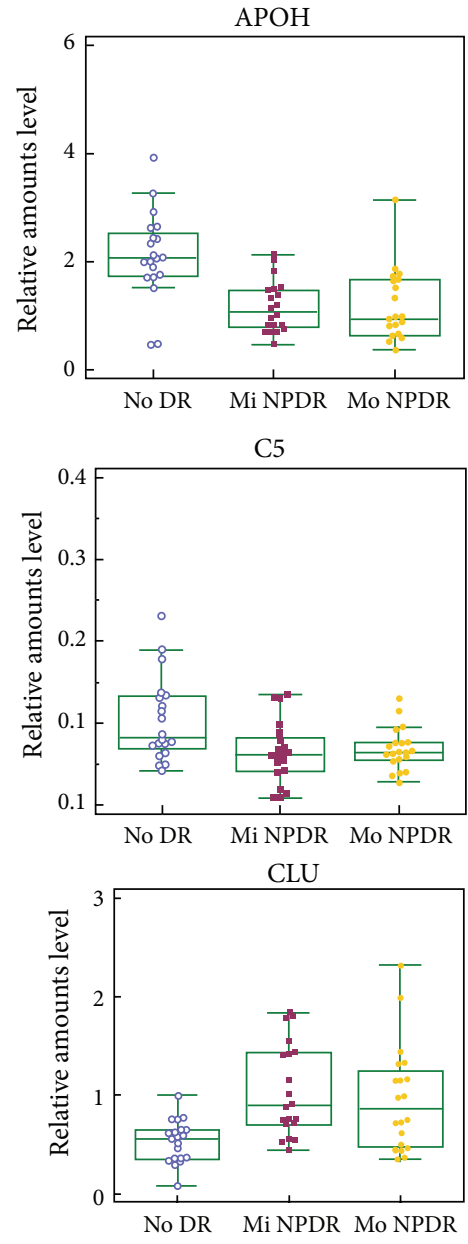

ITIH2

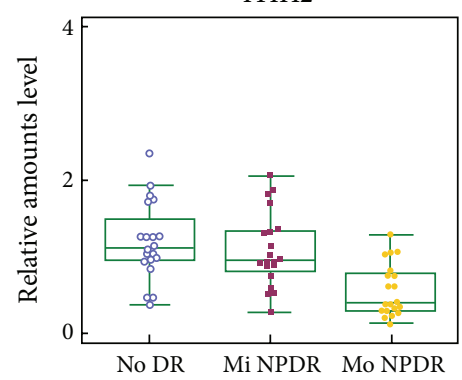



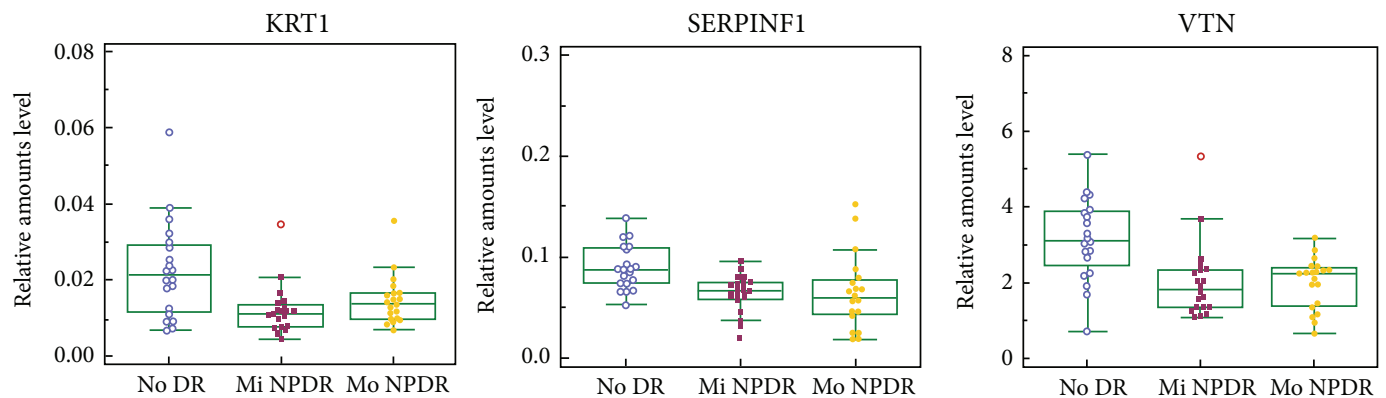

(b)

FIGURE 8: Verification of biomarker candidates using SID-MRM analysis. The 15 selected proteins from our SQ-MRM analysis underwent a second round of verification using SID-MRM analysis in the No DR $(N=20)$, Mi NPDR $(N=20)$, and Mo NPDR $(N=20)$ plasma samples. Box plots (a) and ROC with AUC values (b) were generated based on the SID-MRM analysis.

Classifier: ITIH2

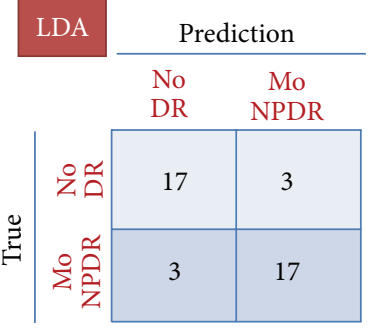

Sensitivity: 0.85 Specificity: 0.85 Error rate: 0.15

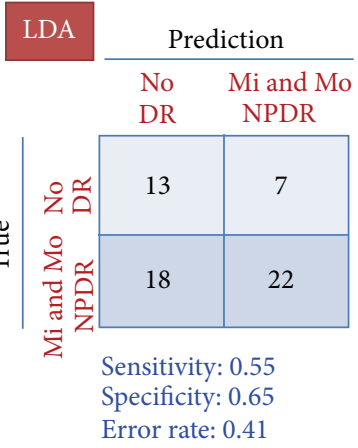

Error rate: 0.41

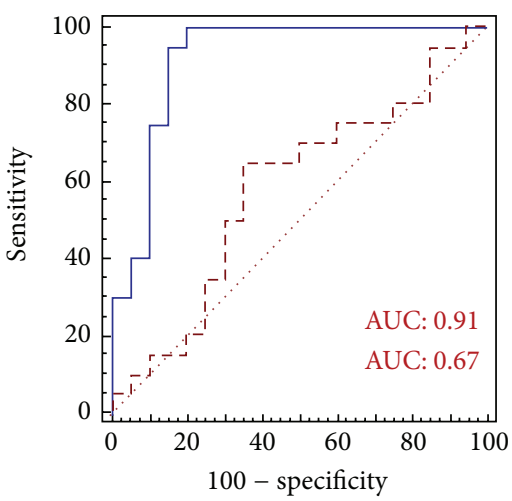

- Mo NPDR

- - Mi and Mo NPDR

(a)

Classifier: APOA4 + C7 + CLU + ITIH2
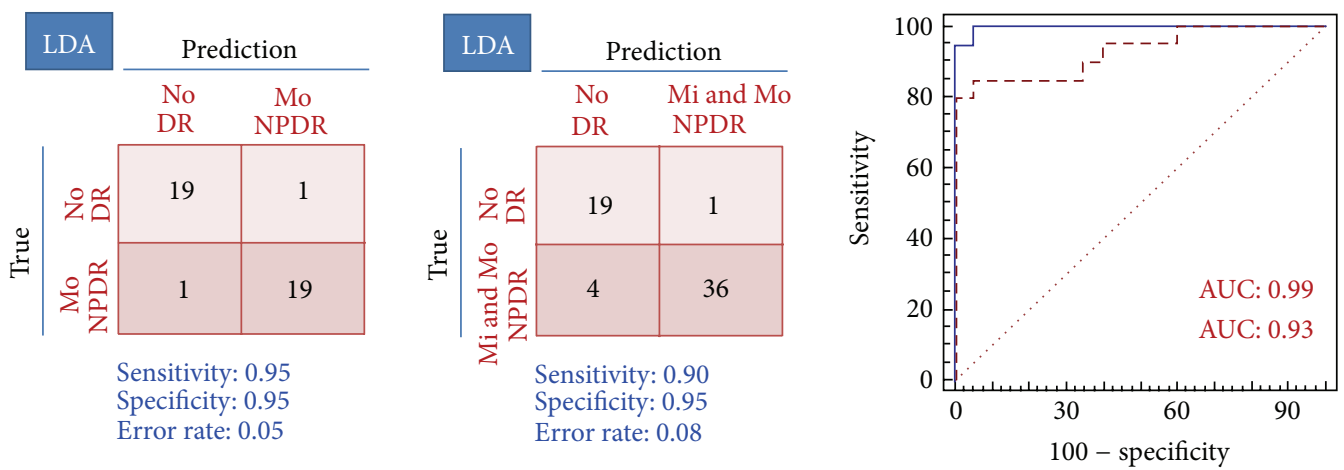

- Mo NPDR

- - - Mi and Mo NPDR

(b)

FIGURE 9: A comparison of the discriminatory power of the 4-marker panel with the best single marker among the No DR, Mo NPDR, and $\mathrm{Mi}+$ Mo NPDR groups. We selected 4 proteins from $t$-test and stepwise MANOVA analyses and used them to construct a 4-marker panel. For comprehensive statistical analysis, LDA algorithms were employed where leave-one-out cross validation (LOOCV) was used to evaluate the discriminatory power among the No DR, Mo NPDR, and Mi + Mo NPDR groups. For comparisons between the best single marker and 4-marker panel, results are presented as confusion matrices. Sensitivity, specificity, error rates, and ROC curves are also represented with AUC values. 


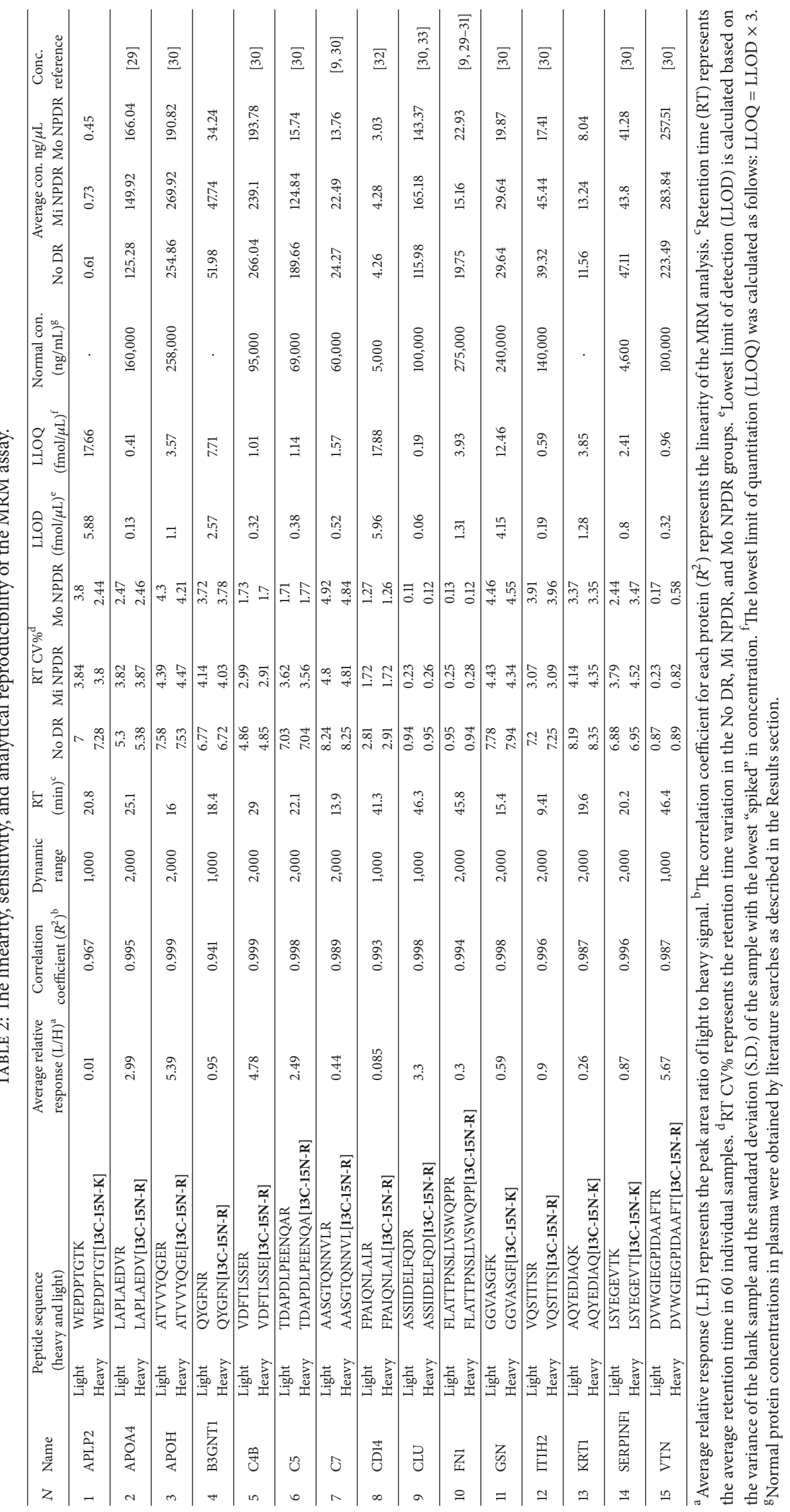


in the No DR, Mi NPDR, and Mo NPDR groups (Table 2 and Supplementary Figure 7). For C5 [36], its concentration in normal plasma was lower in the No DR and Mi NPDR groups, while its level was higher in the Mo NPDR group (Table 2 and Supplementary Figure 7). The normal plasma concentration range of KRT1 has not been previously reported.

In the quantitative analysis using 60 individual samples (Figures 8(a) and 8(b)), the concentrations of 12 proteins (APOA4, APOH, B3GNT1, C4B, C5, C7, CD14, CLU, GSN, ITIH2, KRT1, and VTN) were significantly different in the No DR versus Mi NPDR (AUC value $>0.7$ ) and No DR versus Mo NPDR (AUC value > 0.7) comparisons. By contrast, no significant difference in the concentration of 3 proteins (APLP2, FN1, and SERPINF1) was detected among the No DR, Mi NPDR, and Mo NPDR groups.

4.3. Evaluation of the 4-Marker Panel among the No DR, Mi NPDR, and Mo NPDR Groups. To improve the classification discriminating power between the No DR and NPDR groups, we constructed a multimarker panel and subjected it to statistical evaluation. A similar approach has been carried out to identify a novel biomarker that can distinguish disease status between affected and healthy groups; a multimarker panel that included more than 1 protein showed better performance than a single marker [33,39-42].

Before we selected variables for the multimarker panel, we first considered which combination of sample groups (No DR, Mi NPDR, and Mo NPDR) would show the best discriminating power. Mi NPDR is a very early stage of NPDR and it was not easy to observe differences in the No DR group versus the Mi NPDR group. However, Mo NPDR is a more advanced stage of NPDR and may be more representative of a NPDR diagnosis than Mi NPDR. Thus, the detection of Mo NPDR might be more suitable for NPDR screening. Therefore, we first selected a multimarker panel that showed the best discriminatory power between the No DR and Mo NPDR groups. We then applied this multimarker panel to evaluate its discriminatory power in No DR versus the $\mathrm{Mi}+$ Mo NPDR.

We first determined the correlation and multicollinearity among the 15 potential markers as variables to evaluate performance. Thereafter, we selected 4 potential markers that showed significantly different patterns in the No DR and Mo NPDR groups using Student's $t$-test and stepwise MANOVA. Using the 4 significantly different marker proteins (ITIH2, APOA4, C7, and CLU), the multimarker panel was constructed and statistical validation was performed using LOOCV. The LDA method was employed for statistical analysis of the model.

In a comparison of the No DR group with the Mo NPDR group, the 4-marker panel showed better sensitivity (0.95), specificity (0.95), error rate (5\%), and AUC value (0.99) than the best single marker (ITIH2). Indeed, the single best candidate model showed lower sensitivity $(0.85)$, specificity (0.85), AUC value (0.91), and a higher error rate (15\%). Moreover, for the No DR versus Mi + Mo NPDR comparison, the 4-marker panel (sensitivity, 0.90; specificity, 0.95; error rate, 8\%; and AUC, 0.93 ) also showed better performance than the best single marker (sensitivity, 0.55 ; specificity, 0.65 ; error rate, $41 \%$; and AUC, 0.67$)$. These data demonstrate that the discriminatory power of the 4-marker marker panel was higher than the best single marker model.

Finally, we suggest that the multimarker panel (ITIH2, APOA4, C7, and CLU) is able to distinguish DR status between the patient and normal groups. However, more precise validation with a larger sample size is needed. Further validation in a larger sample size might yield a better understanding of statistical analysis and correlated variables, facilitating the development of better biomarkers for DR.

\section{Conclusion}

We have performed a comprehensive proteomics study to identify biomarkers for DR. We first performed data-mining on the previously published DR-related studies and our experimental data; 96 proteins were then selected. To verify the selected biomarker candidates in plasma, candidates were selected, confirmed, and validated in the plasma of patients in the No DR, Mi NPDR, and Mo NPDR groups using SQ-MRM and SID-MRM analyses. In the final stage, we constructed a model for a multimarker panel using the 11 verified markers derived from our SID-MRM analysis. Our multimarker panel showed merged AUC values of 0.99 (No DR versus Mo NPDR) and 0.93 (No DR versus Mi + Mo NPDR). The 4protein marker panel (APO4, C7, CLU, and ITIH2) can be used as baseline data for the discovery of novel biomarkers for detecting early stage DR and for further validation using a larger panel of proteins.

\section{Conflict of Interests}

The authors declare no competing financial interests.

\section{Authors' Contribution}

Jonghwa Jin and Hophil Min contributed equally to this work.

\section{Acknowledgments}

This work was supported by the Proteogenomic Research Program through the National Research Foundation of Korea and National Research Foundation of Korea (NRF) Grants (nos. 2011-0030740, 2012R1A3A2026438), funded by the Korea government (MSIP). This work was also supported by the Industrial Strategic Technology Development Program (no. 10045352, MKE, Korea) and a grant of the Korea Health Technology R\&D Project through the KHIDI, funded by the Ministry of Health and Welfare, Republic of Korea (no. HI14C1277).

\section{References}

[1] T. A. Ciulla, A. G. Amador, and B. Zinman, "Diabetic retinopathy and diabetic macular edema: pathophysiology, screening, and novel therapies," Diabetes Care, vol. 26, no. 9, pp. 26532664, 2003. 
[2] S. J. Glover, P. I. Burgess, D. B. Cohen et al., "Prevalence of diabetic retinopathy, cataract and visual impairment in patients with diabetes in sub-Saharan Africa," British Journal of Ophthalmology, vol. 96, no. 2, pp. 156-161, 2012.

[3] V. Sundling, C. G. P. Platou, R. W. Jansson, G. Bertelsen, E. Wøllo, and P. Gulbrandsen, "Retinopathy and visual impairment in diabetes, impaired glucose tolerance and normal glucose tolerance: the Nord-Trøndelag Health Study (the HUNT study)," Acta Ophthalmologica, vol. 90, no. 3, pp. 237-243, 2012.

[4] H. Lewis, G. W. Abrams, M. S. Blumenkranz, and R. V. Campo, "Vitrectomy for diabetic macular traction and edema associated with posterior hyaloidal traction," Ophthalmology, vol. 99, no. 5, pp. 753-759, 1992.

[5] G. B. Arden, A. M. P. Hamilton, J. Wilson-Holt, S. Ryan, J. S. Yudkin, and A. Kurtz, "Pattern electroretinograms become abnormal when background diabetic retinopathy deteriorates to a preproliferative stage: possible use as a screening test," British Journal of Ophthalmology, vol. 70, no. 5, pp. 330-335, 1986.

[6] T. Shitama, H. Hayashi, S. Noge et al., "Proteome profiling of vitreoretinal diseases by cluster analysis," Proteomics: Clinical Applications, vol. 2, no. 9, pp. 1265-1280, 2008.

[7] S. Rangasamy, P. G. McGuire, and A. Das, "Diabetic retinopathy and inflammation: novel therapeutic targets," Middle East African Journal of Ophthalmology, vol. 19, no. 1, pp. 52-59, 2012.

[8] S. Gutman and L. G. Kessler, "The US Food and Drug Administration perspective on cancer biomarker development," Nature Reviews Cancer, vol. 6, no. 7, pp. 565-571, 2006.

[9] M. Polanski and N. L. Anderson, "A list of candidate cancer biomarkers for targeted proteomics," Biomarker Insights, vol. 1, pp. 1-48, 2007.

[10] S. Surinova, R. Schiess, R. Hüttenhain, F. Cerciello, B. Wollscheid, and R. Aebersold, "On the development of plasma protein biomarkers," Journal of Proteome Research, vol. 10, no. 1, pp. 5-16, 2011.

[11] S. M. Hewitt, J. Dear, and R. A. Star, "Discovery of protein biomarkers for renal diseases," Journal of the American Society of Nephrology, vol. 15, no. 7, pp. 1677-1689, 2004.

[12] L. R. Bandara, M. D. Kelley, E. A. Lock, and S. Kennedy, "A potential biomarker of kidney damage identified by proteomics: preliminary findings," Biomarkers, vol. 8, no. 4, pp. 272-286, 2003.

[13] J. Jin, Y. H. Ku, Y. Kim et al., "Differential proteome profiling using iTRAQ in microalbuminuric and normoalbuminuric type 2 diabetic patients," Experimental Diabetes Research, vol. 2012, Article ID 168602, 31 pages, 2012.

[14] N. L. Anderson, M. Polanski, R. Pieper et al., "The human plasma proteome: a nonredundant list developed by combination of four separate sources," Molecular - Cellular Proteomics, vol. 3, no. 4, pp. 311-326, 2004.

[15] L. Li, B. Willard, N. Rachdaoui et al., "Plasma proteome dynamics: analysis of lipoproteins and acute phase response proteins with ${ }^{2} \mathrm{H}_{2} \mathrm{O}$ metabolic labeling," Molecular \& Cellular Proteomics, vol. 11, no. 7, article M111.014209, 2012.

[16] L. J. Zimmerman, M. Li, W. G. Yarbrough, R. J. C. Slebos, and D. C. Liebler, "Global stability of plasma proteomes for mass spectrometry-based analyses," Molecular \& Cellular Proteomics, vol. 11, no. 6, article M111.014340, 2012.

[17] N. Rifai, M. A. Gillette, and S. A. Carr, "Protein biomarker discovery and validation: the long and uncertain path to clinical utility," Nature Biotechnology, vol. 24, no. 8, pp. 971-983, 2006.
[18] B.-B. Gao, X. Chen, N. Timothy, L. P. Aiello, and E. P. Feener, "Characterization of the vitreous proteome in diabetes without diabetic retinopathy and diabetes with proliferative diabetic retinopathy," Journal of Proteome Research, vol. 7, no. 6, pp. 2516-2525, 2008.

[19] B.-B. Gao, A. Clermont, S. Rook et al., "Extracellular carbonic anhydrase mediates hemorrhagic retinal and cerebral vascular permeability through prekallikrein activation," Nature Medicine, vol. 13, no. 2, pp. 181-188, 2007.

[20] K. Yamane, A. Minamoto, H. Yamashita et al., "Proteome analysis of human vitreous proteins," Molecular \& Cellular Proteomics, vol. 2, no. 11, pp. 1177-1187, 2003.

[21] A. Decanini, P. R. Karunadharma, C. L. Nordgaard, X. Feng, T. W. Olsen, and D. A. Ferrington, "Human retinal pigment epithelium proteome changes in early diabetes," Diabetologia, vol. 51, no. 6, pp. 1051-1061, 2008.

[22] M. García-Ramírez, F. Canals, C. Hernández et al., "Proteomic analysis of human vitreous fluid by fluorescence-based difference gel electrophoresis (DIGE): a new strategy for identifying potential candidates in the pathogenesis of proliferative diabetic retinopathy," Diabetologia, vol. 50, no. 6, pp. 1294-1303, 2007.

[23] T. Kim, J. K. Sang, K. Kim et al., "Profiling of vitreous proteomes from proliferative diabetic retinopathy and nondiabetic patients," Proteomics, vol. 7, no. 22, pp. 4203-4215, 2007.

[24] K. Kim, S. J. Kim, D. Han et al., "Verification of multimarkers for detection of early stage diabetic retinopathy using multiple reaction monitoring," Journal of Proteome Research, vol. 12, no. 3, pp. 1078-1089, 2013.

[25] H. Wang, L. Feng, J. W. Hu, C. L. Xie, and F. Wang, "Characterisation of the vitreous proteome in proliferative diabetic retinopathy," Proteome Science, vol. 10, no. 1, article 15, 2012.

[26] C. P. Wilkinson, F. L. Ferris III, R. E. Klein et al., "Proposed international clinical diabetic retinopathy and diabetic macular edema disease severity scales," Ophthalmology, vol. 110, no. 9, pp. 1677-1682, 2003.

[27] B. MacLean, D. M. Tomazela, N. Shulman et al., "Skyline: an open source document editor for creating and analyzing targeted proteomics experiments," Bioinformatics, vol. 26, no. 7, pp. 966-968, 2010.

[28] K. Kim, S. J. Kim, H. G. Yu et al., "Verification of biomarkers for diabetic retinopathy by multiple reaction monitoring," Journal of Proteome Research, vol. 9, no. 2, pp. 689-699, 2010.

[29] H. Keshishian, T. Addona, M. Burgess et al., "Quantification of cardiovascular biomarkers in patient plasma by targeted mass spectrometry and stable isotope dilution," Molecular \& Cellular Proteomics, vol. 8, no. 10, pp. 2339-2349, 2009.

[30] B. Schilling, M. J. Rardin, B. X. MacLean et al., "Platformindependent and label-free quantitation of proteomic data using MS1 extracted ion chromatograms in skyline: application to protein acetylation and phosphorylation," Molecular \& Cellular Proteomics, vol. 11, no. 5, pp. 202-214, 2012.

[31] T. A. Addona, S. E. Abbatiello, B. Schilling et al., "Multi-site assessment of the precision and reproducibility of multiple reaction monitoring-based measurements of proteins in plasma," Nature Biotechnology, vol. 27, no. 7, pp. 633-641, 2009.

[32] W. R. Klecka, Discriminant Analysis, SAGE Publications, Beverly Hills, Calif, USA, 1980.

[33] S.-W. Hyung, M. Y. Lee, J.-H. Yu et al., "A serum protein profile predictive of the resistance to neoadjuvant chemotherapy in advanced breast cancers," Molecular \& Cellular Proteomics, vol. 10, no. 10, p. M111.011023, 2011. 
[34] T. A. Addona, X. Shi, H. Keshishian et al., "A pipeline that integrates the discovery and verification of plasma protein biomarkers reveals candidate markers for cardiovascular disease," Nature Biotechnology, vol. 29, no. 7, pp. 635-643, 2011.

[35] D. Domanski, A. J. Percy, J. Yang et al., "MRM-based multiplexed quantitation of 67 putative cardiovascular disease biomarkers in human plasma," Proteomics, vol. 12, no. 8, pp. 1222-1243, 2012.

[36] G. L. Hortin, D. Sviridov, and N. L. Anderson, "High-abundance polypeptides of the human plasma proteome comprising the top 4 logs of polypeptide abundance," Clinical Chemistry, vol. 54, no. 10, pp. 1608-1616, 2008.

[37] N. E. Stathakis, A. Fountas, and E. Tsianos, "Plasma fibronectin in normal subjects and in various disease states," Journal of Clinical Pathology, vol. 34, no. 5, pp. 504-508, 1981.

[38] A. Lutterotti, B. Kuenz, V. Gredler et al., "Increased serum levels of soluble CD14 indicate stable multiple sclerosis," Journal of Neuroimmunology, vol. 181, no. 1-2, pp. 145-149, 2006.

[39] D. Domanski, G. V. C. Freue, L. Sojo et al., "The use of multiplexed MRM for the discovery of biomarkers to differentiate iron-deficiency anemia from anemia of inflammation," Journal of Proteomics, vol. 75, no. 12, pp. 3514-3528, 2012.

[40] H. Loei, H. T. Tan, T. K. Lim et al., "Mining the gastric cancer secretome: identification of GRN as a potential diagnostic marker for early gastric cancer," Journal of Proteome Research, vol. 11, no. 3, pp. 1759-1772, 2012.

[41] P. Kaur, N. M. Rizk, S. Ibrahim et al., "ITRAQ-based quantitative protein expression profiling and MRM verification of markers in type 2 diabetes," Journal of Proteome Research, vol. 11, no. 11, pp. 5527-5539, 2012.

[42] Y.-W. Kim, S. M. Bae, H. Lim, Y. J. Kim, and W. S. Ahn, "Development of multiplexed bead-based immunoassays for the detection of early stage ovarian cancer using a combination of serum biomarkers," PLoS ONE, vol. 7, no. 9, Article ID e44960, 2012. 


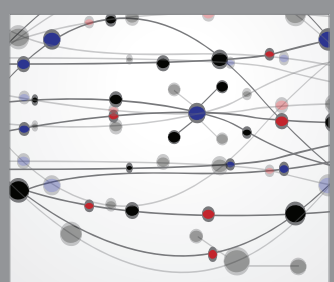

The Scientific World Journal
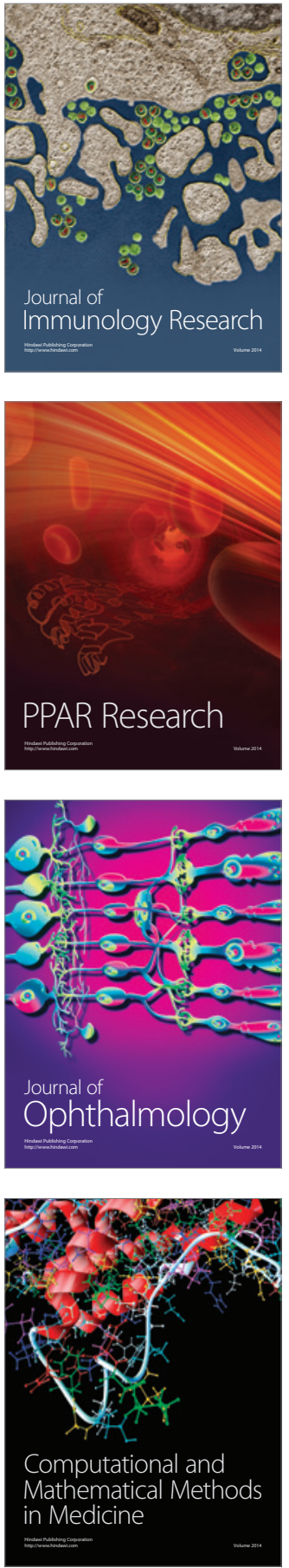

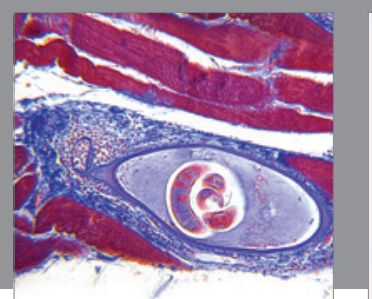

Gastroenterology Research and Practice

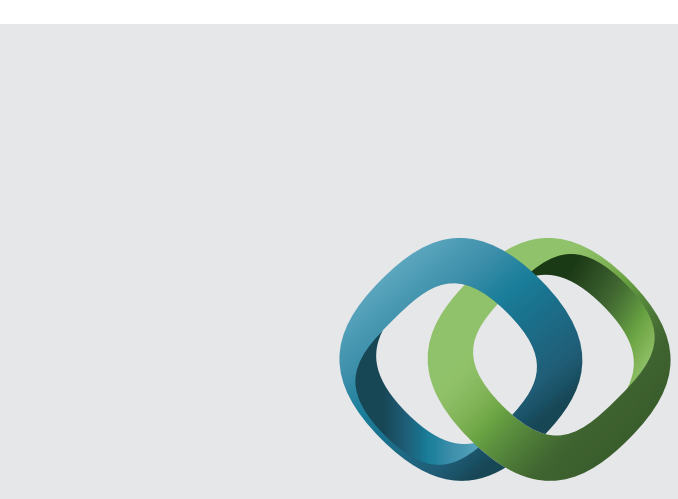

\section{Hindawi}

Submit your manuscripts at

http://www.hindawi.com
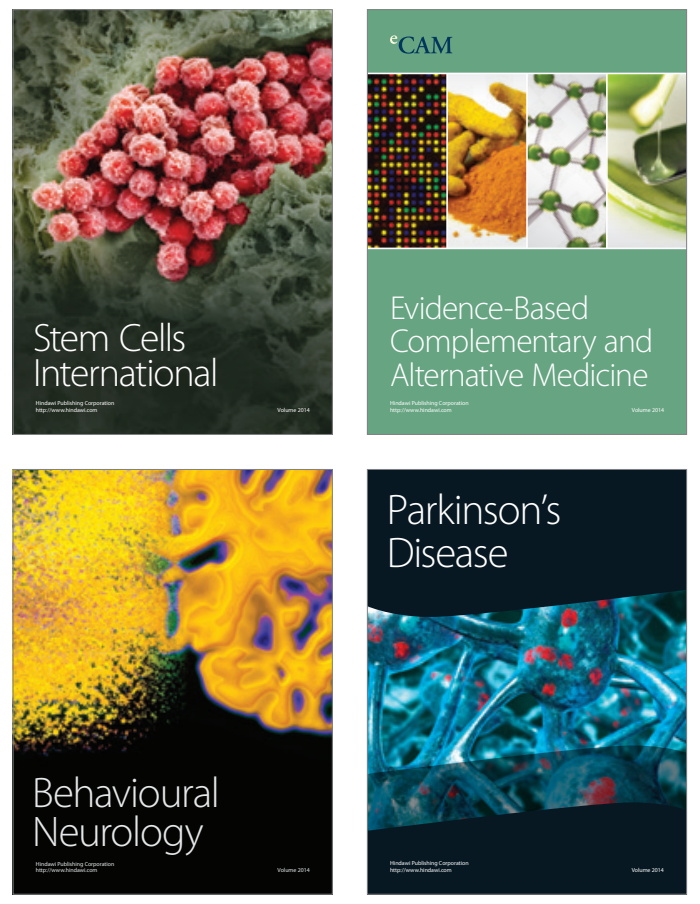
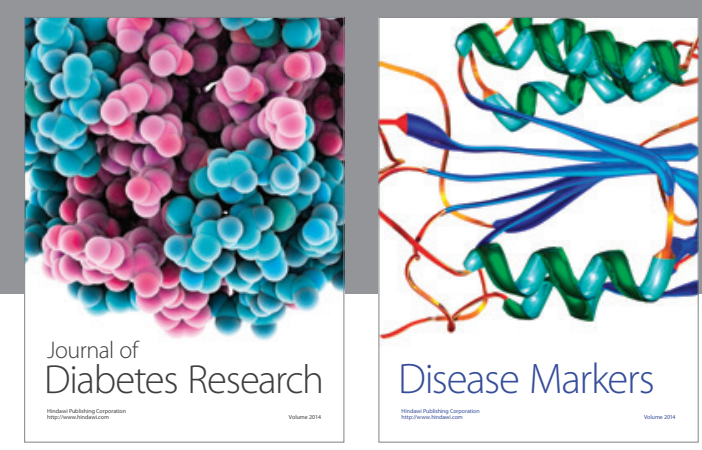

Disease Markers
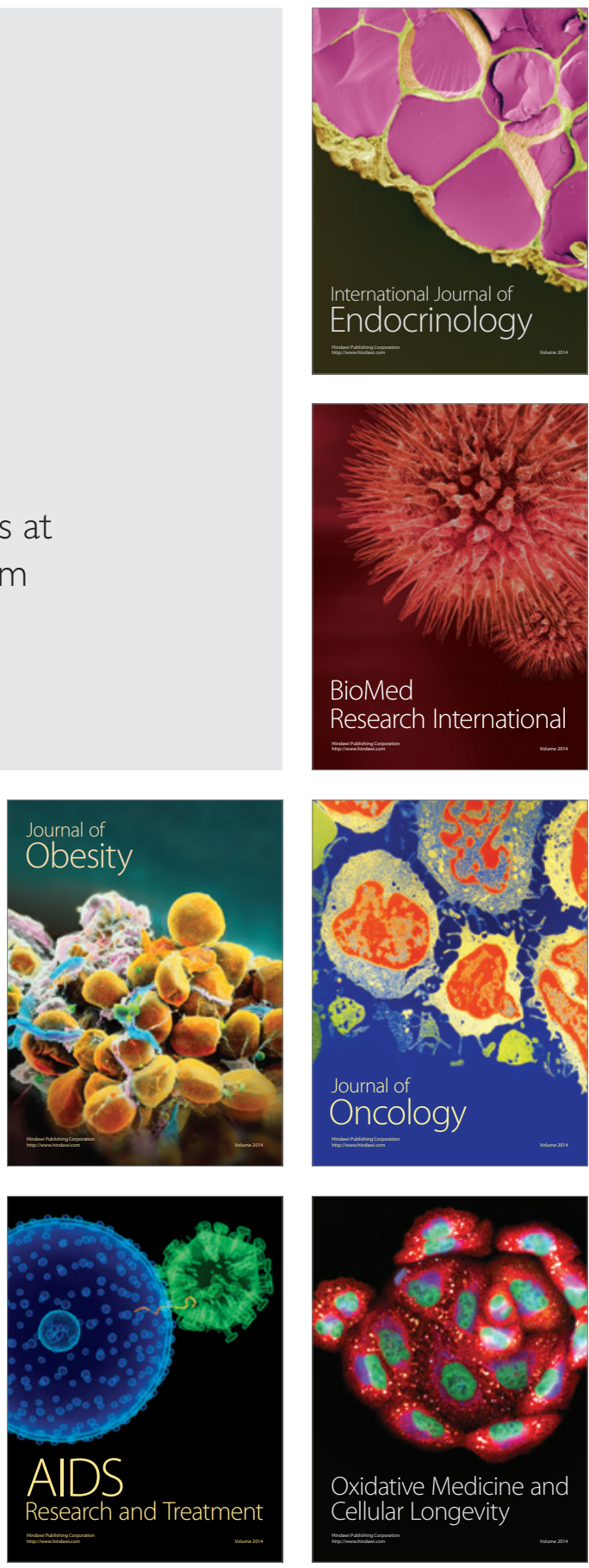